\title{
Understanding the Reactivity of Neutral Geminal Group 14 Element/Phosphorus Frustrated Lewis Pairs
}

\author{
Jorge Juan Cabrera-Trujillo ${ }^{[\mathrm{a}]}$ and Israel Fernández ${ }^{[\mathrm{a}] *}$ \\ Jorge Juan Cabrera-Trujillo and Israel Fernández*
}

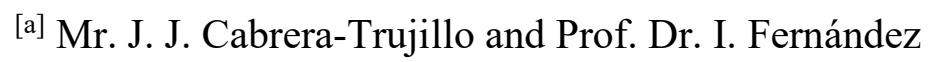

Departamento de Química Orgánica I and Centro de Innovación en Química Avanzada (ORFEO-CINQA)

Facultad de Ciencias Químicas

Universidad Complutense de Madrid, 28040-Madrid, Spain

e-mail: israel@quim.ucm.es

\section{Contents:}

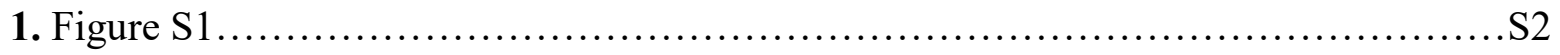

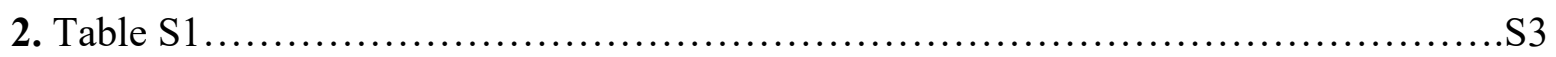

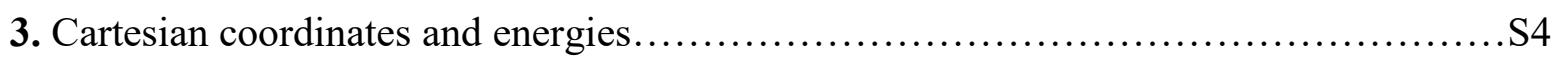




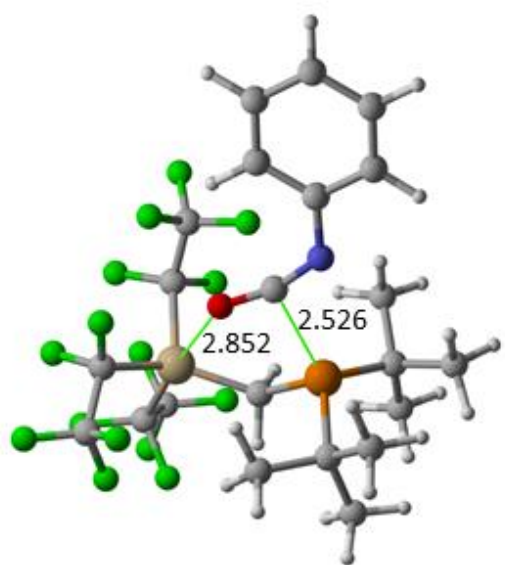

TS-1-Si (PhNCO)

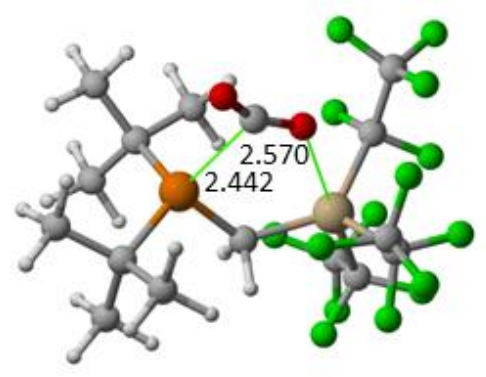

TS-1-Si $\left(\mathrm{CO}_{2}\right)$

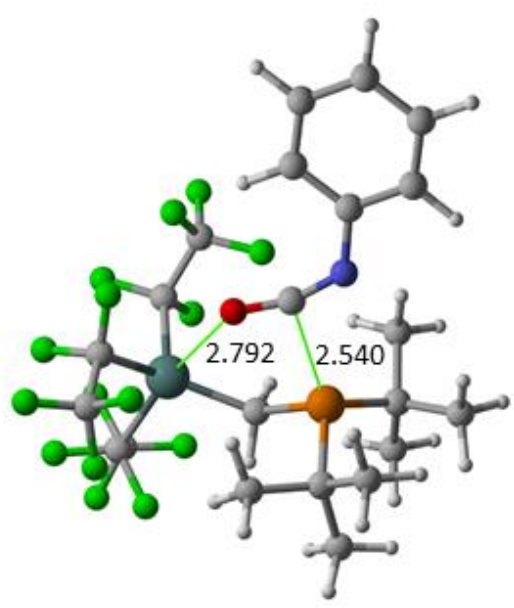

TS-1-Ge (PhNCO)

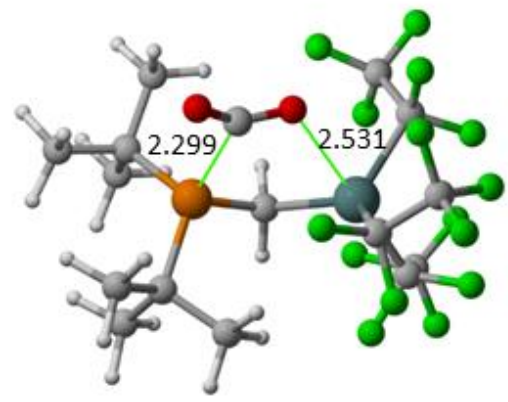

TS-1-Ge $\left(\mathrm{CO}_{2}\right)$

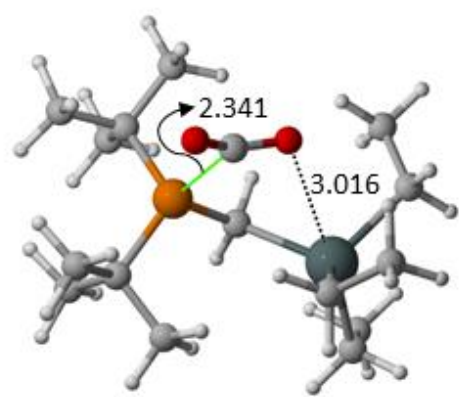

TS-1-Sn' $\left(\mathrm{CO}_{2}\right)$

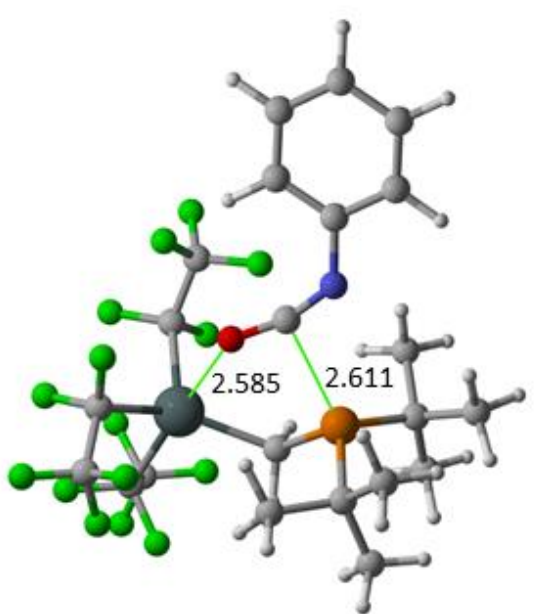

TS-1-Sn (PhNCO)

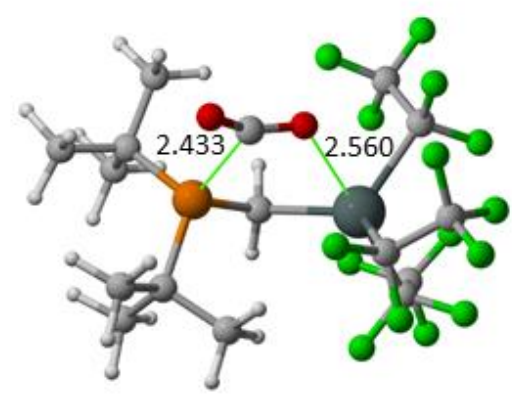

TS-1-Sn $\left(\mathrm{CO}_{2}\right)$

Figure S1. Optimized transition state geometries (M06-2X-def2-SVP level) for the FLP 1-E ( $\mathrm{E}=\mathrm{Si}, \mathrm{Ge}, \mathrm{Sn}$ ) and FLP 1-Sn' mediated $\mathrm{CO}_{2}$ and phenyl isocyanate activation reactions. Bond lengths are given in angstroms. 
Table S1. Effect of the inclusion of relativistic effects in the calculations on the activation barriers.

\begin{tabular}{|c|c|c|}
\hline Reaction & $\Delta \boldsymbol{E}^{\nexists a l}$ & $\Delta \boldsymbol{E}^{\neq b l}$ \\
\hline 1-Si $+\mathbf{C O}_{2}$ & 10.0 & 10.5 \\
\hline 1-Ge $+\mathbf{C O}_{2}$ & 7.6 & 7.8 \\
\hline 1-Sn $+\mathbf{C O}_{2}$ & 6.9 & 6.7 \\
\hline 1-Si + PhNCO & 18.5 & 18.3 \\
\hline 1-Ge + PhNCO & 11.3 & 11.3 \\
\hline 1-Sn + PhNCO & 9.8 & 10.2 \\
\hline
\end{tabular}

Computed activation barriers, $\Delta E^{\neq}=E(T S)-E(R C)$. [a] Computed at the PCM(toluene)-m06-2x/def2TZVPP//m06-2x/def2-SVP level. [b] Computed at the ZORA-COSMO(toluene)-m06-2x/TZ2P//m06-2x/def2-SVP. 
Cartesian coordinates (in $\AA$ ) and total energies (in a.u., noncorrected ZVPE included) of all the stationary points discussed in the text. All calculations have been performed at the M06-2X/def2-SVP level.

$\begin{array}{lr}\mathrm{CO}_{2}: \mathrm{E}= & -188.358325 \\ \mathrm{C} & 0.000000000 \\ \mathrm{O} & 0.000000000 \\ \mathrm{O} & 0.000000000 \\ & \\ \text { PhNCO: } & \mathrm{E}=-399.168574 \\ \mathrm{C} & 0.804474000 \\ \mathrm{C} & -0.086645000 \\ \mathrm{C} & 2.176171000 \\ \mathrm{H} & 2.866305000 \\ \mathrm{C} & 0.402378000 \\ \mathrm{C} & 2.667720000 \\ \mathrm{H} & -0.299679000 \\ \mathrm{H} & 3.742987000 \\ \mathrm{C} & 1.775964000 \\ \mathrm{H} & 2.151251000 \\ \mathrm{H} & 0.404090000 \\ \mathrm{~N} & -1.451364000 \\ \mathrm{C} & -2.579265000 \\ \mathrm{O} & -3.708774000\end{array}$

1-Si: $E=-2709.249201$

P $\quad-2.436886000$

Si $\quad 0.750906000$

F $\quad 1.864997000$

F $\quad 3.256870000$

F 2.612023000

F $\quad 1.859553000$

F $\quad 0.512474000$

$\mathrm{F}$

$\mathrm{F}$

$\mathrm{F}$

\author{
0.000000000 \\ 0.000000000 \\ 0.000000000
}

$-1.278881000$

$-0.200924000$

$-1.041594000$

$-1.886371000$

1.111799000

0.263358000

1.946656000

0.444732000

1. 335935000

2. 360344000

$-2.292617000$

$-0.457612000$

$-0.059940000$

0.231503000

0.003036000

0.030976000

1. 129840000

1.030099000

3. 520147000

3.064992000

3.118682000

0.881050000

1. 442568000

$-1.545932000$

$-1.362318000$

$-0.210032000$

$-2.641748000$

$-1.589534000$

$-1.316033000$

$-3.354237000$

$-2.065341000$

1.293620000

2. 788165000

0.437647000

$-0.696892000$

$-1.659782000$

$-2.116255000$

0.219471000

$-0.388869000$

1. 267086000

1. 506707000

1. 330470000

0.578063000

2. 285443000

1.035653000

1.825839000

2. 011933000

2.748340000

1.032413000

\author{
0.000000000 \\ 1.156136000 \\ $-1.156136000$
}

\author{
0.000004000 \\ 0.000008000 \\ $-0.000014000$ \\ $-0.000021000$ \\ 0.000014000 \\ $-0.000015000$ \\ 0.000027000 \\ $-0.000030000$ \\ 0.000001000 \\ 0.000002000 \\ 0.000011000 \\ 0.000041000 \\ $-0.000008000$ \\ $-0.000028000$
}

0.192247000

$-0.053336000$

$-2.212634000$

$-0.519117000$

$-1.286594000$

0.678879000

$-1.000863000$

2.165434000

2.071123000

2.866030000

2.462127000

4. 047603000

0.331719000

0.488322000

$-2.137087000$

$-1.621963000$

$-2.407714000$

$-0.867358000$

$-0.608961000$

1. 843324000

2.834256000

$-0.211107000$

$-1.628340000$

$-0.880130000$

$-1.797629000$

$-1.216615000$

$-0.398502000$

0.070149000

$-0.528594000$

$-0.041054000$

1. 129912000

$-1.895600000$

$-2.278460000$

$-2.070267000$

$-2.500588000$ 


$\begin{array}{ll}\mathrm{C} & -2.818455000 \\ \mathrm{H} & -2.868195000 \\ \mathrm{H} & -3.366015000 \\ \mathrm{H} & -1.762546000 \\ \mathrm{C} & -3.159342000 \\ \mathrm{C} & -3.674759000 \\ \mathrm{H} & -4.577245000 \\ \mathrm{H} & -3.942298000 \\ \mathrm{C} & -4.286149000 \\ \mathrm{H} & -3.917694000 \\ \mathrm{H} & -4.662591000 \\ \mathrm{H} & -5.135428000 \\ \mathrm{C} & -2.038110000 \\ \mathrm{H} & -1.238973000 \\ \mathrm{H} & -2.456620000 \\ \mathrm{H} & -1.586585000 \\ \mathrm{H} & -2.918683000\end{array}$

1-Ge: $E=-4496.601763$

$\mathrm{Ge}$

C

$\mathrm{H}$

$\mathrm{H}$

C

C
2.687971000

2. 531048000

3. 612480000

2.849769000

$-1.593887000$

$-1.521879000$

$-0.898984000$

$-2.534665000$

$-2.046654000$

$-2.147446000$

$-3.029237000$

$-1.350747000$

$-2.638741000$

$-2.461304000$

$-3.635707000$

$-2.672427000$

$-1.126665000$

$-0.033302000$

$-0.224849000$

0.369020000

$-1.283562000$

1. 376481000

2.771422000

0.220686000

$-1.045095000$

$-1.746305000$

$-2.064379000$

0.038146000

$-1.288437000$

1.757190000

$-1.454521000$

$-1.657335000$

$-2.321032000$

$-0.582900000$

$-2.603728000$

$-2.852761000$

$-2.557279000$

$-3.430666000$

$-1.015139000$

$-1.888805000$

$-0.842020000$

$-0.147316000$

1. 858902000

1. 345927000

2. 919712000

1. 444544000

2. 237357000

2. 231309000

3. 269412000

1. 614897000

2.676489000

2.484524000

3. 725510000

2.581319000

$-1.074667000$

$-3.183987000$

$-2.203447000$

$-2.769744000$

$-1.760298000$

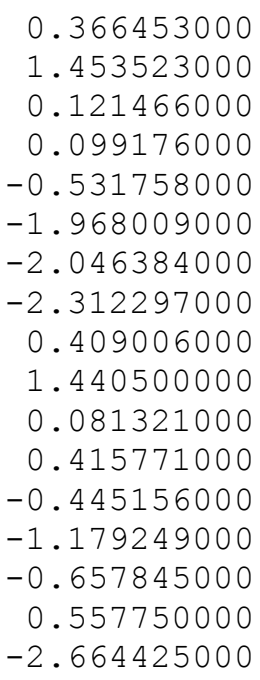

0.046057000

0.885996000

1. 813322000

1.192978000

$-1.333735000$

$-0.779698000$

1. 507685000

2.257856000

$-0.892609000$

$-2.196839000$

$-0.191826000$

0.558874000

0.390513000

2. 080955000

2.466494000

2.353409000

2. 611512000

$-0.082343000$

0.188754000

$-1.179220000$

0.264876000

0.093801000

0.324295000

$-0.992527000$

0.605720000

1.826312000

1.956334000

2. 083684000

2. 552099000

$-0.590613000$

$-1.623067000$

$-0.338504000$

$-0.556910000$

0.235803000

1. 005166000

0.354093000

$-0.761688000$

$-3.063994000$

$-2.732782000$

$-1.946825000$

$-0.039980000$

$-1.169861000$ 


$\begin{array}{ll}F & -0.063771000 \\ F & -2.142620000 \\ F & -2.836561000 \\ F & -1.182300000 \\ F & -0.928588000 \\ F & -3.118087000 \\ F & -1.371220000 \\ F & -3.236014000 \\ F & -1.504276000 \\ F & -3.154946000\end{array}$

1-Sn: $E=-2634.059179$ $\mathrm{Sn}$

C 0.669441000 $-1.167008000$ $-1.260307000$ $-1.037949000$ 0.777324000 0.181822000 1.666640000 1.855205000 2.164271000 2.009129000 $-2.629519000$ $-3.828180000$ $-3.180137000$ $-3.831644000$ $-2.833927000$ $-4.502547000$ $-4.190197000$ $-3.365466000$ $-2.332057000$ $-3.412714000$ $-4.014970000$ $-5.243768000$ $-5.902741000$ $-5.260433000$ $-5.673821000$ $-3.792041000$ $-4.762694000$ $-3.961104000$ $-3.128622000$ $-4.170496000$ $-3.713856000$ $-4.459706000$ $-5.088232000$ $-1.933137000$ $-1.243497000$ $-2.231298000$ $-1.392235000$ 2.347233000 2.738717000 0.720860000 1. 945358000 3.438583000 0.195985000 2.091259000 0.537637000 0.617807000 $-1.142901000$ 2.551262000 0.656129000
1. 550347000

0.914023000

2. 940283000

2.863147000

3. 744967000

$-1.864905000$

$-1.664479000$

$-0.750086000$

1.044808000

0.806764000

$-0.009321000$

0.361437000

$-0.313389000$

1.385547000

0.472914000

$-0.554017000$

$-1.982196000$

$-2.309583000$

1.394613000

2. 825008000

0.347046000

1. 469192000

$-1.465398000$

1. 318986000

1.484602000

2.078359000

0.336439000

2. 892408000

3. 086814000

3.069011000

3. 627528000

1. 271332000

2. 064967000

1. 328337000

0.306719000

$-1.883778000$

$-1.401731000$

$-2.973327000$

$-1.656011000$

$-1.726784000$

$-1.489757000$

$-2.790249000$

$-1.131411000$

$-2.324641000$

$-2.312558000$

$-3.375160000$

$-2.027398000$

2. 895297000

3. 685139000

3.177677000

1. 445202000

1. 013646000

1.662587000

0.570990000

$-0.311569000$

$-1.768377000$

$-0.552051000$

$-1.337768000$

$-2.364714000$
-2.160091000
-2.072822000
-0.861473000
0.508019000
-1.438468000
1.451468000
2.703472000
3.289710000
2.438651000
1.018419000

0.074131000

1.103901000

1. 969114000

1.486016000

$-2.088068000$

$-3.051958000$

0.334918000

1. 815206000

0.932710000

0.419300000

$-0.057368000$

0.899648000

0.087946000

2. 422552000

2.854509000

2.857248000

2.752189000

0.545191000

0.872963000

$-0.538844000$

1. 047118000

0.347134000

0.734818000

$-0.752393000$

0.651980000

1. 424583000

1.604255000

1. 423290000

2. 273051000

$-1.055516000$

$-2.027696000$

$-1.059930000$

$-0.955832000$

$-0.160011000$

0.696121000

$-0.306158000$

$-1.073636000$

$-0.858554000$

1.106892000

0.530672000

2. 272528000

0.731061000

$-2.336965000$

$-2.396371000$

$-4.300552000$

$-2.710639000$

$-2.974982000$

2.396052000

2.404049000 
$\mathrm{Sn}$

C

$\mathrm{H}$

$\mathrm{H}$

C

C

C

C

C

C

P

C

C

C

$\mathrm{H}$

$\mathrm{H}$

$\mathrm{H}$

C

$\mathrm{H}$

$\mathrm{H}$

$\mathrm{H}$

C

$\mathrm{H}$

$\mathrm{H}$

$\mathrm{H}$

C

$\mathrm{H}$

$\mathrm{H}$

$\mathrm{H}$

C
1-Sn': $E=-1146.893045$

\subsection{0 \\ 0.995682000 \\ 2. 889838000}

$-1.786482000$

0.218408000

0.387922000

0.197047000

$-1.810824000$

$-1.217252000$

$-2.563814000$

$-2.386482000$

$-3.041870000$

$-2.647473000$

1. 600246000

2. 919403000

2.132514000

3.028712000

2.067053000

3. 748935000

3.383568000

2.490630000

1. 497160000

2. 455230000

3. 209303000

4.286217000

4.994689000

4.224443000

4.712284000

2.785557000

3.778554000

2. 917191000

2.165051000

3.073302000

2. 590311000

3.333280000

4.008795000

0.857565000

0.201693000

1. 128460000

0.286117000

$-1.271489000$

$-2.862373000$

$-0.133953000$

$-1.360841000$

$-1.675942000$

$-2.080280000$

$-3.632181000$

$-1.324176000$

$-2.893308000$

$-2.795618000$

$-4.091347000$

$-2.974545000$

$-3.266950000$

$-1.598764000$

$-2.752458000$

$E=-1182.931488$

0.492412000

0.765824000
$-3.458424000$

$-3.013601000$

$-1.932249000$

0.056645000

0.469103000

$-0.009282000$

1. 555770000

0.240869000

$-0.928925000$

$-1.846904000$

$-2.008427000$

1. 649264000

3.027219000

0.166184000

1.390681000

$-1.602394000$

1. 547582000

1.830528000

2. 350751000

0.635420000

2.740508000

3.053533000

2. 696233000

3. 523166000

1.026983000

1.854442000

0.855560000

0.128529000

$-1.778649000$

$-1.310822000$

$-2.853573000$

$-1.356681000$

$-2.113031000$

$-2.054446000$

$-3.167027000$

$-1.540344000$

$-2.457263000$

$-2.245619000$

$-3.524971000$

$-2.298801000$

1. 170447000

0.408343000

$-1.007132000$

$-0.803526000$

$-1.891114000$

$-2.673395000$

$-1.882243000$

$-1.979536000$

$-1.203478000$

$-2.962697000$

1. 424557000

1. 628417000

3. 832357000

3. 271477000

3. 080442000

0.150388000

$-0.804652000$
2.004866000

$-0.216464000$

$-0.231526000$

0.023809000

$-0.749925000$

$-1.729197000$

$-0.933213000$

2.189368000

2.974799000

$-0.690618000$

$-2.201556000$

$-0.775588000$

$-0.239936000$

0.452292000

$-0.181185000$

$-0.006158000$

$-1.699713000$

$-2.151485000$

$-1.930293000$

$-2.194821000$

0.417464000

0.059109000

1. 515623000

0.123617000

0.406302000

0.235735000

1. 492462000

$-0.062460000$

$-1.377216000$

$-1.424425000$

$-1.587005000$

$-2.182817000$

1.093695000

2.080699000

0.900316000

1.141304000

0.033746000

$-0.822617000$

$-0.011304000$

0.963161000

2. 432625000

2.474425000

2.797623000

4.059469000

2.696369000

$-0.148397000$

$-0.424836000$

$-2.493918000$

$-2.756542000$

$-2.568275000$

$-0.526937000$

$-1.874947000$

$-0.664918000$

$-0.474674000$

0.854833000

$-1.044210000$

$-1.521056000$ 


\begin{tabular}{|c|c|}
\hline $\mathrm{H}$ & 0.562690000 \\
\hline $\mathrm{P}$ & 1.815938000 \\
\hline B & -1.006775000 \\
\hline C & -1.801575000 \\
\hline C & -2.709876000 \\
\hline $\mathrm{C}$ & -1.610622000 \\
\hline C & -3.381582000 \\
\hline C & -2.302271000 \\
\hline $\mathrm{C}$ & -3.184149000 \\
\hline $\mathrm{C}$ & -1.758643000 \\
\hline $\mathrm{C}$ & -3.161235000 \\
\hline $\mathrm{C}$ & -1.069446000 \\
\hline $\mathrm{C}$ & -3.844443000 \\
\hline C & -1.747514000 \\
\hline $\mathrm{C}$ & -3.137596000 \\
\hline $\mathrm{H}$ & -3.716927000 \\
\hline $\mathrm{H}$ & -2.147447000 \\
\hline $\mathrm{H}$ & -4.067042000 \\
\hline $\mathrm{H}$ & -2.882847000 \\
\hline $\mathrm{H}$ & -0.909383000 \\
\hline $\mathrm{H}$ & 0.019272000 \\
\hline $\mathrm{H}$ & -1.189407000 \\
\hline $\mathrm{H}$ & -3.669363000 \\
\hline $\mathrm{H}$ & -4.930625000 \\
\hline $\mathrm{H}$ & -3.727705000 \\
\hline $\mathrm{C}$ & 3.392554000 \\
\hline $\mathrm{C}$ & 1.691771000 \\
\hline $\mathrm{C}$ & 2.965971000 \\
\hline $\mathrm{H}$ & 2.800977000 \\
\hline $\mathrm{H}$ & 3.823802000 \\
\hline $\mathrm{H}$ & 3.232385000 \\
\hline $\mathrm{C}$ & 0.528158000 \\
\hline $\mathrm{H}$ & 0.714554000 \\
\hline $\mathrm{H}$ & -0.428701000 \\
\hline $\mathrm{H}$ & 0.395844000 \\
\hline $\mathrm{C}$ & 1.405317000 \\
\hline $\mathrm{H}$ & 0.452426000 \\
\hline $\mathrm{H}$ & 2.199234000 \\
\hline $\mathrm{H}$ & 1.327842000 \\
\hline $\mathrm{C}$ & 4.553718000 \\
\hline $\mathrm{H}$ & 4.769473000 \\
\hline $\mathrm{H}$ & 5.468148000 \\
\hline $\mathrm{H}$ & 4.333656000 \\
\hline $\mathrm{C}$ & 3.189325000 \\
\hline $\mathrm{H}$ & 2.806589000 \\
\hline $\mathrm{H}$ & 4.155807000 \\
\hline $\mathrm{H}$ & 2.495236000 \\
\hline $\mathrm{C}$ & 3.751549000 \\
\hline $\mathrm{H}$ & 2.921113000 \\
\hline $\mathrm{H}$ & 4.612438000 \\
\hline $\mathrm{H}$ & 4.036001000 \\
\hline
\end{tabular}

0.949563000

0.631316000

0.148114000

$-1.212897000$

$-1.476739000$

$-2.221326000$

$-2.694473000$

$-3.429490000$

$-3.671561000$

1.492120000

1.570423000

2.674898000

2.765267000

3.867170000

3. 915464000

$-4.622201000$

$-4.189142000$

$-2.880451000$

$-0.714102000$

$-2.055862000$

2.655380000

4. 765474000

4.851671000

2.799282000

0.678587000

0.393856000

$-0.767123000$

$-0.793247000$

$-1.439011000$

$-1.201869000$

0.210972000

$-0.345405000$

0.634458000

$-0.286680000$

$-1.092661000$

$-2.168185000$

$-2.209399000$

$-2.533736000$

$-2.874784000$

1.034025000

0.511909000

1. 005649000

2.085354000

1. 210914000

2. 221203000

1. 321361000

0.718365000

$-1.041963000$

$-1.550629000$

$-1.035350000$

$-1.641712000$
$-1.801015000$

0.196504000

$-0.539354000$

$-0.462781000$

0.578688000

$-1.423815000$

0.668198000

$-1.360609000$

$-0.307521000$

$-0.216187000$

$-0.321115000$

0.117634000

$-0.102016000$

0.358222000

0.246194000

$-0.246398000$

$-2.128745000$

1. 496766000

1. 342902000

$-2.245825000$

0.205870000

0.627636000

0.426340000

$-0.202191000$

$-0.597717000$

$-0.846441000$

1. 475861000

2. 328648000

3.206469000

1.776189000

2.693764000

2.387674000

2. 850895000

1.845630000

3.187211000

0.930400000

0.385220000

0.267234000

1.773997000

$-0.070928000$

0.870236000

$-0.686341000$

0.166774000

$-2.130711000$

$-1.913488000$

$-2.648513000$

$-2.825963000$

$-1.228948000$

$-1.742260000$

$-1.918621000$

$-0.353204000$

\section{Reaction with $\mathrm{CO}_{2}$}

RC-1-Si $\left(\mathrm{CO}_{2}\right): \mathrm{E}=-2897.618126$

$\mathrm{Si}$

$\mathrm{P} \quad 2.573665000$

$\mathrm{F} \quad-0.965712000$

$\mathrm{F} \quad 0.389505000$

$\mathrm{F} \quad-2.081969000$

F

$$
\begin{array}{r}
-0.014719000 \\
0.166843000 \\
1.425684000 \\
2.377496000 \\
3.578376000 \\
3.338101000
\end{array}
$$

-0.158241000
-0.053308000
-2.419405000
-1.003478000
-1.669161000
0.397859000 


\begin{tabular}{|c|c|}
\hline $\mathrm{F}$ & -3.022360000 \\
\hline $\mathrm{F}$ & -2.014744000 \\
\hline $\mathrm{F}$ & -3.141162000 \\
\hline $\mathrm{F}$ & -2.442873000 \\
\hline $\mathrm{F}$ & -1.236659000 \\
\hline $\mathrm{F}$ & -0.384345000 \\
\hline $\mathrm{F}$ & -2.889738000 \\
\hline $\mathrm{F}$ & -0.977310000 \\
\hline $\mathrm{F}$ & -2.913145000 \\
\hline $\mathrm{F}$ & -2.543492000 \\
\hline $\mathrm{F}$ & -0.921435000 \\
\hline O & 0.528360000 \\
\hline O & 2.087025000 \\
\hline C & -0.780524000 \\
\hline C & -1.888034000 \\
\hline $\mathrm{C}$ & -1.902601000 \\
\hline C & -1.492013000 \\
\hline C & -1.724301000 \\
\hline C & -2.044934000 \\
\hline C & 0.957138000 \\
\hline $\mathrm{H}$ & 0.929643000 \\
\hline $\mathrm{H}$ & 0.978377000 \\
\hline C & 3.604782000 \\
\hline C & 3.230762000 \\
\hline $\mathrm{H}$ & 3.501794000 \\
\hline $\mathrm{H}$ & 3.776252000 \\
\hline $\mathrm{H}$ & 2.155142000 \\
\hline C & 3.361611000 \\
\hline $\mathrm{H}$ & 2.334345000 \\
\hline $\mathrm{H}$ & 4.031978000 \\
\hline $\mathrm{H}$ & 3.566985000 \\
\hline C & 5.091942000 \\
\hline $\mathrm{H}$ & 5.461488000 \\
\hline $\mathrm{H}$ & 5.677126000 \\
\hline $\mathrm{H}$ & 5.293374000 \\
\hline $\mathrm{C}$ & 3.115048000 \\
\hline C & 3.404778000 \\
\hline $\mathrm{H}$ & 4.298105000 \\
\hline $\mathrm{H}$ & 3.590526000 \\
\hline $\mathrm{H}$ & 2.558256000 \\
\hline C & 4.353140000 \\
\hline $\mathrm{H}$ & 4.160405000 \\
\hline $\mathrm{H}$ & 4.599593000 \\
\hline $\mathrm{H}$ & 5.235912000 \\
\hline $\mathrm{C}$ & 1.980248000 \\
\hline $\mathrm{H}$ & 1.061950000 \\
\hline $\mathrm{H}$ & 2.293742000 \\
\hline $\mathrm{H}$ & 1.746002000 \\
\hline C & 1.320549000 \\
\hline
\end{tabular}

2.036166000

1. 271600000

$-0.315574000$

$-1.043465000$

$-2.114311000$

$-0.489133000$

$-0.545794000$

$-1.246444000$

$-2.659759000$

$-3.234175000$

$-3.206502000$

1. 782624000

3. 462093000

1.699851000

2.692758000

0.055648000

$-0.915930000$

$-1.136425000$

$-2.591804000$

$-0.782862000$

$-1.099127000$

$-1.711143000$

$-0.829260000$

$-0.223472000$

0.842665000

$-0.748925000$

$-0.313091000$

$-2.340942000$

$-2.590846000$

$-2.785343000$

$-2.836182000$

$-0.557334000$

$-1.088209000$

$-0.910901000$

0.517831000

$-0.264672000$

$-1.738828000$

$-2.091666000$

$-1.874792000$

$-2.389535000$

0.585046000

1. 651153000

0.469062000

0.286968000

0.212474000

$-0.377962000$

0.100246000

1. 272871000

2. 614453000
$-0.493182000$

1. 975052000

1.016749000

3.431670000

1. 997689000

3.125458000

$-1.742471000$

$-2.543736000$

$-0.035377000$

$-2.070789000$

$-0.650965000$

1. 690305000

1. 373652000

$-1.109063000$

$-0.706865000$

1.412182000

2.528449000

$-1.411719000$

$-1.028004000$

0.116408000

1.169844000

$-0.476268000$

1.199676000

2.562949000

2.608928000

3. 363367000

2. 781708000

1. 249542000

1. 550760000

2. 003577000

0.292623000

0.948775000

0.060215000

1. 812880000

0.823102000

$-1.816226000$

$-2.096187000$

$-1.562062000$

$-3.174219000$

$-1.827848000$

$-2.141827000$

$-1.950991000$

$-3.209483000$

$-1.562645000$

$-2.731989000$

$-2.610898000$

$-3.782298000$

$-2.564717000$

1.509655000

RC-1-Ge $\left(\mathrm{CO}_{2}\right): E=-4684.976168$

$\begin{array}{lr}\text { Ge } & 0.678075000 \\ \mathrm{P} & -2.642490000 \\ \mathrm{~F} & 0.946391000 \\ \mathrm{~F} & -0.458077000 \\ \mathrm{~F} & 2.009303000 \\ \mathrm{~F} & 1.446394000 \\ \mathrm{~F} & 2.946547000 \\ \mathrm{~F} & 1.985033000 \\ \mathrm{~F} & 3.142420000\end{array}$

$-0.022787000$

0.146427000

0.068531000

0.120580000

2.488663000

2. 380760000

1.145872000

1.758163000

3. 602282000

3.369953000

2. 075717000

1.364688000

$-0.304374000$

0.558120000

$-1.992046000$

$-1.101816000$ 


\begin{tabular}{|c|c|}
\hline $\mathrm{F}$ & 2.414264000 \\
\hline $\mathrm{F}$ & 1.228292000 \\
\hline $\mathrm{F}$ & 0.361106000 \\
\hline $\mathrm{F}$ & 2.921095000 \\
\hline $\mathrm{F}$ & 1.045493000 \\
\hline $\mathrm{F}$ & 2.918593000 \\
\hline F & 2.590044000 \\
\hline F & 0.941071000 \\
\hline O & -0.592725000 \\
\hline 0 & -2.175555000 \\
\hline $\mathrm{C}$ & 0.720197000 \\
\hline $\mathrm{C}$ & 1.809185000 \\
\hline $\mathrm{C}$ & 1.899365000 \\
\hline C & 1.477685000 \\
\hline $\mathrm{C}$ & 1.753529000 \\
\hline $\mathrm{C}$ & 2.070894000 \\
\hline C & -1.056080000 \\
\hline $\mathrm{H}$ & -1.025338000 \\
\hline $\mathrm{H}$ & -1.063145000 \\
\hline C & -3.728922000 \\
\hline $\mathrm{C}$ & -3.368539000 \\
\hline $\mathrm{H}$ & -3.615933000 \\
\hline $\mathrm{H}$ & -3.942785000 \\
\hline $\mathrm{H}$ & -2.299968000 \\
\hline $\mathrm{C}$ & -3.524913000 \\
\hline $\mathrm{H}$ & -2.507833000 \\
\hline $\mathrm{H}$ & -4.216932000 \\
\hline $\mathrm{H}$ & -3.727858000 \\
\hline $\mathrm{C}$ & -5.201806000 \\
\hline $\mathrm{H}$ & -5.562981000 \\
\hline $\mathrm{H}$ & -5.818019000 \\
\hline $\mathrm{H}$ & -5.371378000 \\
\hline $\mathrm{C}$ & -3.150425000 \\
\hline $\mathrm{C}$ & -3.497758000 \\
\hline $\mathrm{H}$ & -4.422326000 \\
\hline $\mathrm{H}$ & -3.656044000 \\
\hline $\mathrm{H}$ & -2.690916000 \\
\hline C & -4.339406000 \\
\hline $\mathrm{H}$ & -4.100159000 \\
\hline $\mathrm{H}$ & -4.573980000 \\
\hline $\mathrm{H}$ & -5.244462000 \\
\hline $\mathrm{C}$ & -1.965951000 \\
\hline $\mathrm{H}$ & -1.096617000 \\
\hline $\mathrm{H}$ & -2.261719000 \\
\hline $\mathrm{H}$ & -1.659950000 \\
\hline C & -1.396755000 \\
\hline
\end{tabular}

\begin{tabular}{|c|c|}
\hline-0.914150000 & -3.535905000 \\
\hline-2.024286000 & -2.114824000 \\
\hline-0.365839000 & -3.181267000 \\
\hline-0.612361000 & 1.714682000 \\
\hline-1.363125000 & 2.549258000 \\
\hline-2.630154000 & -0.080745000 \\
\hline-3.343088000 & 1.920378000 \\
\hline-3.225149000 & 0.536354000 \\
\hline 1.847126000 & -1.604203000 \\
\hline 3.490502000 & -1.224143000 \\
\hline 1.714144000 & 1.197256000 \\
\hline 2.721656000 & 0.792533000 \\
\hline 0.126838000 & -1.475992000 \\
\hline-0.810924000 & -2.614174000 \\
\hline-1.206365000 & 1.401023000 \\
\hline-2.633470000 & 0.933052000 \\
\hline-0.843388000 & -0.199509000 \\
\hline-1.073336000 & -1.274243000 \\
\hline-1.810955000 & 0.325481000 \\
\hline-0.757891000 & -1.223993000 \\
\hline-0.070600000 & -2.551739000 \\
\hline 1.001694000 & -2.519673000 \\
\hline-0.528642000 & -3.373253000 \\
\hline-0.169677000 & -2.799790000 \\
\hline-2.268140000 & -1.378228000 \\
\hline-2.519846000 & -1.710700000 \\
\hline-2.646086000 & -2.148774000 \\
\hline-2.819414000 & -0.451997000 \\
\hline-0.464683000 & -0.919252000 \\
\hline-1.036019000 & -0.052590000 \\
\hline-0.752944000 & -1.786164000 \\
\hline 0.606239000 & -0.727050000 \\
\hline-0.417377000 & 1.812731000 \\
\hline-1.894158000 & 1.99471000 \\
\hline-2.167542000 & 1.46732900 \\
\hline-2.101332000 & 3.065846000 \\
\hline-2.558529000 & 1.64922700 \\
\hline 0.457894000 & 2.23667800 \\
\hline 1.526339000 & 2.131128000 \\
\hline 0.262992000 & 3.295552000 \\
\hline 0.249802000 & 1.651816000 \\
\hline-0.061682000 & 2.720924000 \\
\hline-0.711543000 & 2.55102600 \\
\hline-0.198639000 & 3.77335600 \\
\hline 0.986652000 & 2.597100000 \\
\hline 2.660043000 & -1.39187100 \\
\hline
\end{tabular}

RC-1-Sn $\left(\mathrm{CO}_{2}\right): E=-2822.436086$

$\begin{array}{lrrr}\text { Sn } & 0.644939000 & 0.111030000 & -0.072080000 \\ \text { P } & -2.707761000 & -0.089177000 & -0.132872000 \\ \text { F } & 0.983062000 & -0.491166000 & -2.926950000 \\ F & -0.435682000 & -1.911966000 & -2.075330000 \\ F & 2.102743000 & -2.815866000 & -2.946251000 \\ F & 1.492208000 & -3.278583000 & -0.936337000 \\ F & 2.942499000 & -1.715862000 & -1.292901000 \\ F & 2.265038000 & -1.980215000 & 1.391873000 \\ F & 3.220524000 & -0.016616000 & 1.394489000 \\ F & 2.401012000 & -0.693054000 & 3.830723000 \\ F & 1.177935000 & 0.890267000 & 3.023344000 \\ F & 0.411102000 & -1.120095000 & 3.123019000\end{array}$




$\begin{array}{ll}\mathrm{F} & 3.077062000 \\ \mathrm{~F} & 1.307338000 \\ \mathrm{~F} & 2.205054000 \\ \mathrm{~F} & 2.493595000 \\ \mathrm{~F} & 0.498511000 \\ \mathrm{O} & -0.537973000 \\ \mathrm{O} & -2.091286000 \\ \mathrm{C} & 0.722068000 \\ \mathrm{C} & 1.838674000 \\ \mathrm{C} & 2.035036000 \\ \mathrm{C} & 1.507807000 \\ \mathrm{C} & 1.786085000 \\ \mathrm{C} & 1.761805000 \\ \mathrm{C} & -1.251859000 \\ \mathrm{H} & -1.224566000 \\ \mathrm{H} & -1.302580000 \\ \mathrm{C} & -4.010065000 \\ \mathrm{C} & -3.701776000 \\ \mathrm{H} & -3.806196000 \\ \mathrm{H} & -4.407638000 \\ \mathrm{H} & -2.684723000 \\ \mathrm{C} & -3.982686000 \\ \mathrm{H} & -3.011205000 \\ \mathrm{H} & -4.743455000 \\ \mathrm{H} & -4.206980000 \\ \mathrm{C} & -5.403046000 \\ \mathrm{H} & -5.730843000 \\ \mathrm{H} & -6.135579000 \\ \mathrm{H} & -5.435123000 \\ \mathrm{C} & -3.066965000 \\ \mathrm{C} & -3.586967000 \\ \mathrm{H} & -4.591682000 \\ \mathrm{H} & -3.649975000 \\ \mathrm{H} & -2.916468000 \\ \mathrm{C} & -4.060902000 \\ \mathrm{H} & -3.670624000 \\ \mathrm{H} & -4.224255000 \\ \mathrm{H} & -5.037279000 \\ \mathrm{C} & -1.739746000 \\ \mathrm{H} & -1.045479000 \\ \mathrm{H} & -1.926802000 \\ \mathrm{H} & -1.249988000 \\ \mathrm{H} & \\ \mathrm{H} & -1.326297000\end{array}$

1.469951000

2. 213311000

2. 737454000

4.050380000

3. 483609000

$-2.338212000$

$-3.741788000$

$-1.250253000$

$-2.298407000$

$-0.655804000$

$-0.397715000$

1. 827273000

3. 060840000

0.654045000

0.217362000

1. 748734000

$-0.043822000$

$-1.292633000$

$-2.210485000$

$-1.354039000$

$-1.267355000$

1. 184881000

1. 302695000

1. 064716000

2.114591000

$-0.211436000$

0.693640000

$-0.409376000$

$-1.058810000$

1. 245135000

2.578652000

2. 483164000

3. 305342000

3.005095000

0.653620000

$-0.279096000$

1. 372743000

0.436711000

1. 494756000

2.095424000

2. 070143000

0.556049000

$-3.024970000$
-1.104879000
-2.141756000
1.172362000
-0.514563000
0.084216000
0.733358000
-0.251189000
-1.838692000
-1.759192000
1.492398000
2.905266000
-0.937063000
-0.039359000
0.763244000
1.772590000
0.869037000
1.250693000
2.093616000
1.495003000
2.937548000
2.517509000
2.163617000
2.664896000
2.952405000
1.626635000
0.634713000
0.103736000
1.433813000
-0.067678000
-1.427335000
-0.890937000
-0.457027000
-1.717509000
-0.128810000
-2.437128000
-2.870640000
-3.255794000
-1.983574000
-2.158424000
-1.553717000
-3.078862000
-2.467756000
0.220980000

0.024850000

$-0.212668000$

0.061347000

$-1.674868000$

$-2.010373000$

$-2.247103000$

1.321407000

2.614814000

0.024996000

1. 229195000

0.920978000

1. 703295000

1.428882000

1. 025532000

1.398650000

0.513834000 


$\begin{array}{ll}\mathrm{H} & -3.345113000 \\ \mathrm{H} & -1.618620000 \\ \mathrm{C} & -3.061147000 \\ \mathrm{H} & -2.110709000 \\ \mathrm{H} & -3.835634000 \\ \mathrm{H} & -3.323595000 \\ \mathrm{C} & -4.348941000 \\ \mathrm{H} & -4.732462000 \\ \mathrm{H} & -5.081971000 \\ \mathrm{H} & -4.303836000 \\ \mathrm{C} & -2.100320000 \\ \mathrm{C} & -2.736755000 \\ \mathrm{H} & -3.735203000 \\ \mathrm{H} & -2.851761000 \\ \mathrm{H} & -2.113172000 \\ \mathrm{C} & -3.036490000 \\ \mathrm{H} & -2.572300000 \\ \mathrm{H} & -3.249945000 \\ \mathrm{H} & -3.996168000 \\ \mathrm{C} & -0.803962000 \\ \mathrm{H} & -0.140631000 \\ \mathrm{H} & -1.049558000 \\ \mathrm{H} & -0.252967000 \\ \mathrm{C} & -0.205198000 \\ \mathrm{H} & 3.316557000 \\ \mathrm{H} & 3.912520000 \\ \mathrm{H} & 2.982675000 \\ \mathrm{H} & 1.427122000 \\ \mathrm{H} & 1.975466000 \\ \mathrm{H} & 3.665129000 \\ \mathrm{H} & 2.290233000 \\ \mathrm{H} & 2.587589000 \\ \mathrm{H} & 2.369028000 \\ \mathrm{H} & 1.027850000 \\ \mathrm{H} & 0.716048000 \\ \mathrm{H} & 1.944825000 \\ \mathrm{H} & 2.715004000 \\ \mathrm{H} & 2.574102000 \\ \mathrm{H} & 3.784293000 \\ & \end{array}$

$$
\begin{array}{r}
-2.448906000 \\
-2.132433000 \\
0.213851000 \\
0.193896000 \\
-0.209264000 \\
1.261934000 \\
-0.635916000 \\
0.375976000 \\
-1.149147000 \\
-1.178898000 \\
1.567396000 \\
2.593505000 \\
2.281688000 \\
3.554415000 \\
2.777136000 \\
1.282332000 \\
0.577288000 \\
2.219892000 \\
0.856391000 \\
2.167058000 \\
2.528916000 \\
3.031582000 \\
1.442376000 \\
-2.940432000 \\
-1.805178000 \\
-0.352130000 \\
-1.961821000 \\
-1.984081000 \\
-0.503924000 \\
2.245191000 \\
2.836881000 \\
4.162987000 \\
2.671424000 \\
3.324774000 \\
-0.806711000 \\
-1.370595000 \\
-2.452241000 \\
-1.260614000
\end{array}
$$

RC-2 $\left(\mathrm{CO}_{2}\right): E=-1371.294622$

$\begin{array}{lrrr}\mathrm{C} & 0.416087000 & -0.920159000 & 2.954841000 \\ \mathrm{O} & -0.683534000 & -0.572561000 & 2.867335000 \\ \mathrm{C} & 0.272819000 & 0.059473000 & -1.573641000 \\ \mathrm{H} & 0.467314000 & -0.835388000 & -2.176548000 \\ \mathrm{H} & 0.503187000 & 0.949296000 & -2.176835000 \\ \mathrm{O} & 1.504928000 & -1.285489000 & 3.098029000 \\ \mathrm{~B} & -1.141988000 & 0.073683000 & -0.885343000 \\ \mathrm{P} & 1.402462000 & 0.005363000 & -0.058337000 \\ \mathrm{C} & 2.569401000 & -1.437498000 & -0.499281000 \\ \mathrm{C} & 3.801303000 & -1.391434000 & 0.413425000 \\ \mathrm{H} & 3.519704000 & -1.241760000 & 1.466457000 \\ \mathrm{H} & 4.337316000 & -2.351700000 & 0.339432000 \\ \mathrm{H} & 4.503052000 & -0.599311000 & 0.115620000 \\ \mathrm{C} & 1.769234000 & -2.702917000 & -0.151967000 \\ \mathrm{H} & 0.820418000 & -2.758539000 & -0.705090000 \\ \mathrm{H} & 2.363290000 & -3.595008000 & -0.410482000 \\ \mathrm{H} & 1.540938000 & -2.744516000 & 0.922368000 \\ \mathrm{C} & 3.025395000 & -1.532502000 & -1.958275000 \\ \mathrm{H} & 3.570794000 & -0.642069000 & -2.293262000\end{array}$

2.134134000

1. 848554000

2. 310277000

2.862294000

2. 972003000

2.121030000

0.330193000

0.137189000

0.974365000

$-0.626949000$

$-0.999087000$

$-0.061104000$

0.275263000

$-0.590309000$

0.827428000

$-2.181474000$

$-2.886955000$

$-2.720810000$

$-1.860515000$

$-1.562819000$

$-0.763268000$

$-2.200842000$

$-2.183694000$

$-0.809249000$

0.741183000

1. 545582000

3.252034000

2. 403675000

3.214629000

0.012792000

$-0.916827000$

1. 248385000

2.181040000

1. 228368000

$-2.240516000$

$-2.688271000$

$-3.281527000$

$-1.578334000$

$-2.060493000$

2.954841000

2.867335000

$-1.573641000$

.176548000

2.176835000

3. 098029000

$-0.885343000$

$-0.058337000$

0.499281000

0.413425000

1. 466457000

0.339432000

0.115620000

$-0.151967000$

$-0.705090000$

$-0.410482000$

$-1.958275000$

$-2.293262000$ 


$\begin{array}{lr}\mathrm{H} & 3.702906000 \\ \mathrm{H} & 2.187750000 \\ \mathrm{C} & 2.355036000 \\ \mathrm{C} & 3.298228000 \\ \mathrm{H} & 3.699463000 \\ \mathrm{H} & 4.153742000 \\ \mathrm{H} & 2.780656000 \\ \mathrm{C} & 1.317845000 \\ \mathrm{H} & 0.588738000 \\ \mathrm{H} & 1.843707000 \\ \mathrm{H} & 0.756213000 \\ \mathrm{C} & 3.136969000 \\ \mathrm{H} & 3.934758000 \\ \mathrm{H} & 3.603313000 \\ \mathrm{H} & 2.466802000 \\ \mathrm{C} & -1.936419000 \\ \mathrm{C} & -2.951261000 \\ \mathrm{C} & -1.692242000 \\ \mathrm{C} & -3.657262000 \\ \mathrm{H} & -3.194579000 \\ \mathrm{C} & -2.407893000 \\ \mathrm{H} & -0.934708000 \\ \mathrm{C} & -3.386592000 \\ \mathrm{H} & -4.430404000 \\ \mathrm{H} & -2.201881000 \\ \mathrm{H} & -3.944901000 \\ \mathrm{C} & -1.754219000 \\ \mathrm{C} & -2.005941000 \\ \mathrm{C} & -2.034738000 \\ \mathrm{C} & -2.506000000 \\ \mathrm{H} & -1.774400000 \\ \mathrm{C} & -2.557271000 \\ \mathrm{H} & -1.835279000 \\ \mathrm{C} & -2.787961000 \\ \mathrm{H} & -2.676713000 \\ \mathrm{H} & -2.772893000 \\ \mathrm{H} & -3.183544000 \\ \mathrm{H} & \end{array}$

$\begin{array}{rr}-2.396504000 & -2.061190000 \\ -1.701298000 & -2.649154000 \\ 1.648333000 & -0.158335000 \\ 1.786411000 & -1.352904000 \\ 2.813099000 & -1.385882000 \\ 1.100837000 & -1.282320000 \\ 1.601937000 & -2.307082000 \\ 2.778708000 & -0.211414000 \\ 2.709405000 & 0.609428000 \\ 3.743061000 & -0.120814000 \\ 2.795155000 & -1.155560000 \\ 1.813197000 & 1.153783000 \\ 1.069951000 & 1.271655000 \\ 2.811775000 & 1.175536000 \\ 1.733149000 & 2.022766000 \\ -1.265039000 & -0.645524000 \\ -1.359651000 & 0.324377000 \\ -2.405129000 & -1.432951000 \\ -2.543480000 & 0.526312000 \\ -0.487377000 & 0.933263000 \\ -3.586902000 & -1.254976000 \\ -2.366127000 & -2.219256000 \\ -3.660409000 & -0.264260000 \\ -2.593576000 & 1.294764000 \\ -4.451723000 & -1.887873000 \\ -4.586333000 & -0.114356000 \\ 1.462573000 & -0.456922000 \\ 1.825373000 & 0.876108000 \\ 2.409488000 & -1.455687000 \\ 3.087498000 & 1.197093000 \\ 1.122513000 & 1.678832000 \\ 3.663297000 & -1.142665000 \\ 2.165382000 & -2.503544000 \\ 4.008367000 & 0.188731000 \\ 3.352992000 & 2.242012000 \\ 4.378463000 & -1.938582000 \\ 4.994149000 & 0.439354000\end{array}$

TS-1-Si $\left(\mathrm{CO}_{2}\right): \mathrm{E}=-2897.601948$

$\begin{array}{lrrr}\mathrm{P} & 2.439236000 & -0.166248000 & 0.161773000 \\ \mathrm{Si} & -0.819004000 & -0.060303000 & -0.307251000 \\ \mathrm{~F} & -2.638550000 & 1.851126000 & -1.001753000 \\ \mathrm{~F} & -1.267574000 & 1.182379000 & -2.578802000 \\ \mathrm{~F} & -0.914800000 & 3.654533000 & -2.195242000 \\ \mathrm{~F} & 0.745859000 & 2.613635000 & -1.303906000 \\ \mathrm{~F} & -0.788537000 & 3.510417000 & -0.061967000 \\ \mathrm{~F} & -3.139992000 & -0.266628000 & 0.850407000 \\ \mathrm{~F} & -1.627399000 & -1.424765000 & 1.926249000 \\ \mathrm{~F} & -2.005436000 & 2.034297000 & 1.954480000 \\ \mathrm{~F} & -0.851796000 & 0.727422000 & 3.248080000 \\ \mathrm{~F} & -2.986380000 & 0.589443000 & 3.208516000 \\ \mathrm{~F} & -3.041526000 & -0.840717000 & -1.804059000 \\ \mathrm{~F} & -1.104310000 & -1.499237000 & -2.571953000 \\ \mathrm{~F} & -0.927962000 & -3.191794000 & -0.342442000 \\ \mathrm{~F} & -3.012173000 & -2.769048000 & 0.023711000 \\ \mathrm{~F} & -2.400864000 & -3.568005000 & -1.871551000 \\ \mathrm{O} & 0.484525000 & 1.570841000 & 1.190426000 \\ \mathrm{O} & 2.548084000 & 2.492170000 & 1.633191000 \\ \mathrm{C} & -1.354711000 & 1.549498000 & -1.268881000 \\ \mathrm{C} & -0.564612000 & 2.867637000 & -1.189346000 \\ \mathrm{C} & -1.870713000 & -0.232585000 & 1.331645000\end{array}$




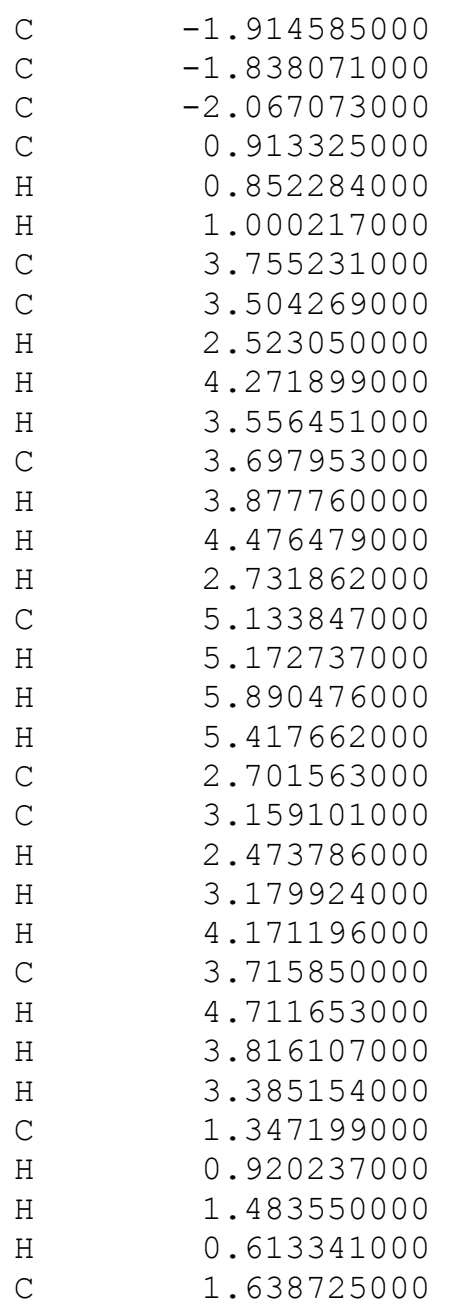

$$
\begin{array}{r}
0.815255000 \\
-1.319063000 \\
-2.741626000 \\
-0.643752000 \\
-1.735545000 \\
-0.241580000 \\
-0.113055000 \\
1.207987000 \\
1.227007000 \\
1.334029000 \\
2.069968000 \\
-1.272984000 \\
-2.246416000 \\
-1.123877000 \\
-1.316794000 \\
-0.044492000 \\
0.743101000 \\
0.192390000 \\
-1.001630000 \\
-1.566277000 \\
-2.887260000 \\
-3.234344000 \\
-3.662695000 \\
-2.813869000 \\
-1.082151000 \\
-0.912291000 \\
-1.846016000 \\
-0.145375000 \\
-1.773128000 \\
-0.829756000 \\
-2.439582000 \\
-2.251795000 \\
1.842894000
\end{array}
$$

2.460930000 $-1.437608000$ $-0.894764000$ $-0.747674000$ $-0.871603000$ $-1.772741000$ $-1.183709000$ $-1.927806000$ $-2.424690000$ $-2.707447000$ $-1.246618000$ $-2.181348000$ $-1.708429000$ $-2.946969000$ $-2.704815000$ $-0.517525000$

0.251059000 $-1.281947000$ $-0.057332000$ 1. 392213000 0.773687000 $-0.014942000$ 1. 556373000 0.352458000 2. 438272000 2.009029000 3.225338000 2. 910724000 2.084353000 2.459934000 2. 950304000 1. 420881000 1.295809000

TS-1-Ge $\left(\mathrm{CO}_{2}\right): \mathrm{E}=-4684.962582$

0.090834000

0.055062000

1. 521025000

$-0.030812000$

$-0.216714000$

$-1.897503000$

$-0.609040000$

$-2.503574000$

$-1.017695000$

$-2.869650000$

$-0.951790000$

$-2.527482000$

1. 768185000

2.808513000

1.104563000

3.200890000

1.891908000

$-1.884277000$

$-2.181358000$

0.243199000

$-0.650714000$

$-1.458913000$

$-1.978741000$

1. 729146000

1. 985106000

0.469144000 


$\begin{array}{ll}\mathrm{H} & -0.902532000 \\ \mathrm{H} & -0.938196000 \\ \mathrm{C} & -3.655208000 \\ \mathrm{C} & -3.119004000 \\ \mathrm{H} & -3.216953000 \\ \mathrm{H} & -3.711365000 \\ \mathrm{H} & -2.066234000 \\ \mathrm{C} & -3.664301000 \\ \mathrm{H} & -2.658570000 \\ \mathrm{H} & -4.291518000 \\ \mathrm{H} & -4.078220000 \\ \mathrm{C} & -5.073678000 \\ \mathrm{H} & -5.566701000 \\ \mathrm{H} & -5.682320000 \\ \mathrm{H} & -5.069586000 \\ \mathrm{C} & -3.177259000 \\ \mathrm{C} & -3.798333000 \\ \mathrm{H} & -4.718155000 \\ \mathrm{H} & -4.062782000 \\ \mathrm{H} & -3.096111000 \\ \mathrm{C} & -4.198373000 \\ \mathrm{H} & -3.766925000 \\ \mathrm{H} & -4.494224000 \\ \mathrm{H} & -5.107834000 \\ \mathrm{C} & -1.994724000 \\ \mathrm{H} & -1.207489000 \\ \mathrm{H} & -2.368449000 \\ \mathrm{H} & -1.551101000 \\ \mathrm{C} & -1.846280000\end{array}$

$$
\begin{array}{r}
-1.802131000 \\
-1.225475000 \\
-1.367113000 \\
-1.664906000 \\
-0.790422000 \\
-2.483033000 \\
-1.979697000 \\
-2.663602000 \\
-3.098697000 \\
-3.404209000 \\
-2.528653000 \\
-0.800018000 \\
-0.673220000 \\
-1.502530000 \\
0.167375000 \\
0.703260000 \\
-0.329963000 \\
-0.768196000 \\
0.169013000 \\
-1.143215000 \\
1.789400000 \\
2.513699000 \\
2.330239000 \\
1.373224000 \\
1.377098000 \\
0.669196000 \\
1.848441000 \\
2.169175000 \\
1.196975000
\end{array}
$$

TS-1-Sn $\left(\mathrm{CO}_{2}\right): \mathrm{E}=-2822.425358$

Sn
P
F
F
F
F
F
F
F
F
F
F
F
F
F
F
F
O
O
C
C
C
C
C
C
C
H
H
C
C

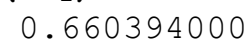

$-2.683953000$

1.231185000

$-0.251032000$

2.391832000

1. 536139000

3.006188000

1.887279000

3.132344000

2.053158000

1.247821000

0.071243000

2. 970308000

1.037210000

3.033132000

2.721692000

1.072603000

$-0.755596000$

$-2.769325000$

0.868602000

1. 972663000

1.860205000

1.312197000

1.829374000

2.185256000

$-1.187147000$

$-1.133630000$

$-1.176663000$

$-3.913146000$

$-3.437172000$
0.067616000

$-0.015831000$

2. 505866000

2. 986428000

4. 010119000

2. 939410000

1. 979461000

0.260278000

$-0.855882000$

$-2.434009000$

$-2.837741000$

$-1.756922000$

0.172855000

$-0.268180000$

$-2.339597000$

$-2.321677000$

$-2.734989000$

1.011921000

2.079653000

2. 267296000

2.828261000

$-0.627633000$

$-1.942714000$

$-0.528468000$

$-2.009611000$

$-1.005681000$

$-1.844682000$

$-1.426497000$

$-1.257924000$

$-1.518033000$
-0.152954000
1.517289000
-0.645676000
-2.055731000
-2.715053000
-2.494207000
-2.053775000
0.170181000
0.264315000
-0.351200000
1.176563000
-0.781352000
0.192311000
-1.371903000
-1.306821000
1.661138000
2.603592000
2.194248000
3.549572000
2.842110000
1.290127000
0.583578000
2.202784000
0.838110000
2.371612000
2.666935000
3.293874000
1.757659000
-1.765189000

0.090615000

0.039295000

1. 622726000

0.096373000

$-0.141453000$

$-1.801968000$

$-0.547335000$

$-2.648232000$

$-1.251029000$

$-3.165834000$

$-1.203187000$

$-2.645497000$

2.054062000

2.986598000

1.032996000

3.163846000

1. 835500000

$-1.821518000$

$-2.127030000$

0.343548000

$-0.560835000$

$-1.639389000$

$-2.193123000$

1. 904968000

1.995632000

0.404500000

$-0.305677000$

1. 420459000

$-0.661561000$

$-2.100581000$ 


$\begin{array}{ll}\mathrm{H} & -3.507178000 \\ \mathrm{H} & -4.081068000 \\ \mathrm{H} & -2.400683000 \\ \mathrm{C} & -3.963140000 \\ \mathrm{H} & -2.980677000 \\ \mathrm{H} & -4.655873000 \\ \mathrm{H} & -4.322249000 \\ \mathrm{C} & -5.302860000 \\ \mathrm{H} & -5.750103000 \\ \mathrm{H} & -5.971141000 \\ \mathrm{H} & -5.267195000 \\ \mathrm{C} & -3.218649000 \\ \mathrm{C} & -3.834219000 \\ \mathrm{H} & -4.797571000 \\ \mathrm{H} & -4.018068000 \\ \mathrm{H} & -3.163825000 \\ \mathrm{C} & -4.207696000 \\ \mathrm{H} & -3.780021000 \\ \mathrm{H} & -4.438975000 \\ \mathrm{H} & -5.153625000 \\ \mathrm{C} & -1.961501000 \\ \mathrm{H} & -1.224443000 \\ \mathrm{H} & -2.254139000 \\ \mathrm{H} & -1.483709000 \\ \mathrm{C} & -1.881120000\end{array}$

$$
\begin{array}{r}
-0.606946000 \\
-2.284055000 \\
-1.884710000 \\
-2.583907000 \\
-3.076725000 \\
-3.267386000 \\
-2.462973000 \\
-0.614791000 \\
-0.506598000 \\
-1.256843000 \\
0.374711000 \\
0.696769000 \\
-0.329238000 \\
-0.708242000 \\
0.151250000 \\
-1.184858000 \\
1.838018000 \\
2.571780000 \\
2.355054000 \\
1.475519000 \\
1.299020000 \\
0.538276000 \\
1.833896000 \\
2.035239000 \\
1.413649000
\end{array}
$$

TS-1-Sn' $\quad\left(\mathrm{CO}_{2}\right): E=-1335.252529$

$\begin{array}{lrrr}\mathrm{Sn} & 1.830730000 & -0.190969000 & 0.001672000 \\ \mathrm{P} & -1.564179000 & -0.018283000 & -0.006364000 \\ \mathrm{O} & 0.081310000 & 2.152115000 & 0.739480000 \\ \mathrm{O} & -1.730616000 & 1.989377000 & 2.141284000 \\ \mathrm{C} & 1.745045000 & -0.044113000 & 2.171953000 \\ \mathrm{C} & 2.577358000 & 1.124027000 & 2.702219000 \\ \mathrm{C} & 2.815771000 & 1.533172000 & -0.881089000 \\ \mathrm{C} & 2.019273000 & 2.178876000 & -2.014021000 \\ \mathrm{C} & 2.923401000 & -1.988991000 & -0.575620000 \\ \mathrm{C} & 3.060341000 & -2.122369000 & -2.093289000 \\ \mathrm{C} & -0.113146000 & -0.422164000 & -1.027526000 \\ \mathrm{H} & -0.076151000 & 0.313532000 & -1.845702000 \\ \mathrm{H} & -0.195875000 & -1.421158000 & -1.484292000 \\ \mathrm{C} & -2.863070000 & 0.670626000 & -1.192458000 \\ \mathrm{C} & -2.382084000 & 2.089053000 & -1.543577000 \\ \mathrm{H} & -2.385405000 & 2.746540000 & -0.662130000 \\ \mathrm{H} & -3.065817000 & 2.523168000 & -2.290127000 \\ \mathrm{H} & -1.369125000 & 2.095439000 & -1.973333000 \\ \mathrm{C} & -3.002573000 & -0.133146000 & -2.488083000 \\ \mathrm{H} & -2.043195000 & -0.208407000 & -3.020875000 \\ \mathrm{H} & -3.713053000 & 0.380865000 & -3.156063000 \\ \mathrm{H} & -3.382141000 & -1.147999000 & -2.317693000 \\ \mathrm{C} & -4.210736000 & 0.791021000 & -0.472825000 \\ \mathrm{H} & -4.682208000 & -0.187622000 & -0.305242000 \\ \mathrm{H} & -4.898934000 & 1.387966000 & -1.092596000 \\ \mathrm{H} & -4.098576000 & 1.299564000 & 0.497266000 \\ \mathrm{C} & -2.127346000 & -1.630436000 & 0.790698000 \\ \mathrm{C} & -2.860522000 & -2.577100000 & -0.161554000 \\ \mathrm{H} & -3.826382000 & -2.170047000 & -0.489479000 \\ \mathrm{H} & -3.062407000 & -3.526828000 & 0.360335000 \\ \mathrm{H} & -2.258037000 & -2.807346000 & -1.053359000 \\ \mathrm{C} & -3.018666000 & -1.274631000 & 1.989861000 \\ \mathrm{H} & -2.509778000 & -0.576700000 & 2.670454000 \\ \mathrm{H} & -3.264908000 & -2.193273000 & 2.546166000\end{array}$

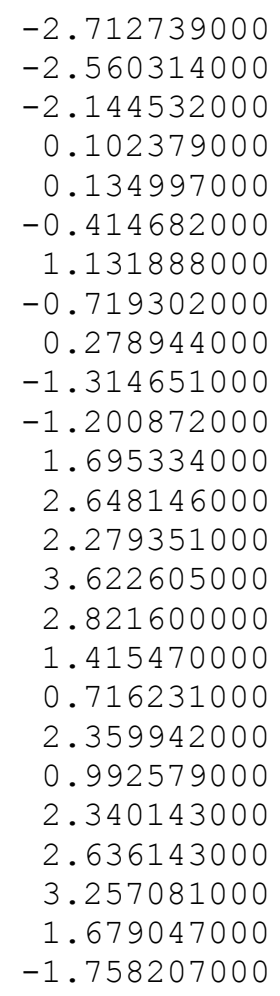

0.001672000
-0.006364000
0.739480000
2.141284000
2.171953000
2.702219000
-0.881089000
-2.014021000
-0.575620000
-2.093289000
-1.027526000
-1.845702000
-1.484292000
-1.192458000
-1.543577000
-0.662130000
-2.290127000
-1.973333000
-2.488083000
-3.020875000
-3.156063000
-2.317693000
-0.472825000
-0.305242000
-1.092596000
0.497266000
0.790698000
-0.161554000
-0.489479000
0.360335000
-1.053359000
1.989861000
2.670454000
2.546166000 


$\begin{array}{lr}\mathrm{H} & -3.962639000 \\ \mathrm{C} & -0.873613000 \\ \mathrm{H} & -0.211258000 \\ \mathrm{H} & -1.185514000 \\ \mathrm{H} & -0.297731000 \\ \mathrm{C} & -0.920868000 \\ \mathrm{H} & 2.975351000 \\ \mathrm{H} & 3.812266000 \\ \mathrm{H} & 2.534596000 \\ \mathrm{H} & 1.035476000 \\ \mathrm{H} & 1.853327000 \\ \mathrm{H} & 3.916827000 \\ \mathrm{H} & 2.414630000 \\ \mathrm{H} & 3.631379000 \\ \mathrm{H} & 3.576767000 \\ \mathrm{H} & 2.077237000 \\ \mathrm{H} & 0.697580000 \\ \mathrm{H} & 2.095603000 \\ \mathrm{H} & 2.564677000 \\ \mathrm{H} & 2.180945000 \\ \mathrm{H} & 3.631690000 \\ & \end{array}$

$$
\begin{array}{r}
-0.808470000 \\
-2.344746000 \\
-2.674074000 \\
-3.243359000 \\
-1.713384000 \\
1.817866000 \\
2.259132000 \\
1.219096000 \\
3.060066000 \\
2.512893000 \\
1.480407000 \\
-1.951355000 \\
-2.869177000 \\
-3.017473000 \\
-1.252838000 \\
-2.192407000 \\
0.074448000 \\
-1.001854000 \\
1.163345000 \\
2.080886000 \\
1.055523000
\end{array}
$$

TS-2 $\left(\mathrm{CO}_{2}\right): E=-1371.282585$

C
$\mathrm{O}$
$\mathrm{C}$
$\mathrm{H}$
$\mathrm{H}$
$\mathrm{O}$
$\mathrm{B}$
$\mathrm{P}$
$\mathrm{C}$
$\mathrm{C}$
$\mathrm{H}$
$\mathrm{H}$
$\mathrm{H}$
$\mathrm{C}$
$\mathrm{H}$
$\mathrm{H}$
$\mathrm{H}$
$\mathrm{C}$
$\mathrm{H}$
$\mathrm{H}$
$\mathrm{H}$
$\mathrm{C}$
$\mathrm{C}$
$\mathrm{H}$
$\mathrm{H}$
$\mathrm{H}$
$\mathrm{C}$
$\mathrm{H}$
$\mathrm{H}$
$\mathrm{H}$
$\mathrm{C}$
$\mathrm{C}$
$\mathrm{C}$
$\mathrm{C}$
$\mathrm{C}$

$$
0.648073000
$$

$-0.474985000$

0.190639000

0.197983000

0.378351000

1.497232000

$-1.218883000$

1.613912000

2.470009000

3.807256000

3.693196000

4.185393000

4.567930000

1. 541974000

0.511493000

1. 930405000

1.501594000

2.693914000

3. 317136000

3. 208650000

1.748944000

2.724186000

3. 504923000

4.030094000

4. 265114000

2.842067000

1. 812610000

1.165436000

2.443561000

1.165024000

3. 681752000

4. 422569000

4.230313000

3.131865000

$-2.377727000$

$-3.641851000$

$-2.214301000$

$-4.686075000$
$-0.205274000$

$-0.485039000$

$-0.365348000$

$-1.367215000$

0.351435000

0.039735000

$-0.003336000$

$-0.255417000$

$-1.929015000$

$-1.888830000$

$-1.455301000$

$-2.917436000$

$-1.315904000$

$-2.919115000$

$-2.894066000$

$-3.941627000$

$-2.710729000$

$-2.408368000$

$-1.718432000$

$-3.382729000$

$-2.558490000$

1. 169240000

0.924713000

1. 850421000

0.140064000

0.644697000

2. 391220000

2. 558957000

3. 284320000

2. 307933000

1. 465707000

0.670921000

2.395906000

1.597814000

$-1.058766000$

$-0.677228000$

$-2.401347000$

$-1.592374000$
1.678912000

1. 316048000

0.501340000

1.871486000

2.007042000

1.296330000

$-0.070396000$

$-1.230207000$

$-2.426705000$

$-1.650850000$

$-2.850315000$

$-0.100375000$

$-0.150307000$

$-2.385978000$

$-2.529940000$

$-2.585951000$

2.488841000

2. 588945000

3. 802212000

2. 331178000

2. 391070000

2.189500000

1. 911051000

$-1.158194000$

$-1.604468000$

$-1.975208000$

2.970959000

$-0.497220000$

0.026124000

$-0.132848000$

0.615962000

1.621433000

0.729119000

0.066841000

0.591297000

0.205879000

0.458890000

1.670275000

$-1.570597000$

$-2.153048000$

$-1.543399000$

$-2.111729000$

$-0.504302000$

$-1.795379000$

$-2.082964000$

$-1.673280000$

$-2.628786000$

$-0.681642000$

0.193887000

$-0.814953000$

$-1.564802000$

0.661267000

0.810375000

0.443053000

1. 604320000

$-0.392920000$

0.091725000

$-0.777460000$

0.204242000 


$\begin{array}{ll}\mathrm{H} & -3.807671000 \\ \mathrm{C} & -3.251700000 \\ \mathrm{H} & -1.255725000 \\ \mathrm{C} & -4.491436000 \\ \mathrm{H} & -5.656177000 \\ \mathrm{H} & -3.096063000 \\ \mathrm{H} & -5.307359000 \\ \mathrm{C} & -1.523977000 \\ \mathrm{C} & -1.810229000 \\ \mathrm{C} & -1.542423000 \\ \mathrm{C} & -2.068514000 \\ \mathrm{H} & -1.818593000 \\ \mathrm{C} & -1.821223000 \\ \mathrm{H} & -1.336160000 \\ \mathrm{C} & -2.073964000 \\ \mathrm{H} & -2.270571000 \\ \mathrm{H} & -1.833529000 \\ \mathrm{H} & -2.278996000\end{array}$

0.361731000

$-3.324455000$

$-2.741732000$

$-2.919844000$

$-1.269443000$

$-4.360759000$

$-3.639865000$

1. 527427000

2. 091756000

2. 382109000

3. 456406000

1. 451778000

3. 742157000

1.978379000

4.286452000

3.873571000

4.379840000

5.352990000
0.383973000

$-0.671359000$

$-1.175527000$

$-0.176023000$

0.585713000

$-0.975673000$

$-0.090490000$

$-0.244339000$

1.009365000

$-1.359993000$

1.143734000

1.893071000

$-1.235126000$

$-2.356567000$

0.023312000

2.132032000

$-2.120975000$

0.129841000

3-Si $\left(\mathrm{CO}_{2}\right): E=-2897.623224$

$\begin{array}{lrrr}\mathrm{P} & 2.339923000 & -0.359712000 & -0.091206000 \\ \mathrm{Si} & -0.723356000 & 0.094383000 & -0.218966000 \\ \mathrm{~F} & -2.649737000 & 2.012684000 & -0.492010000 \\ \mathrm{~F} & -1.349597000 & 1.774863000 & -2.242191000 \\ \mathrm{~F} & -1.074900000 & 4.115378000 & -1.239045000 \\ \mathrm{~F} & 0.684988000 & 2.948988000 & -0.822224000 \\ \mathrm{~F} & -0.728145000 & 3.407432000 & 0.754287000 \\ \mathrm{~F} & -3.096661000 & -0.403898000 & 0.928629000 \\ \mathrm{~F} & -1.575478000 & -1.843235000 & 1.559110000 \\ \mathrm{~F} & -1.827425000 & 1.499653000 & 2.501321000 \\ \mathrm{~F} & -0.552460000 & -0.078937000 & 3.265396000 \\ \mathrm{~F} & -2.683358000 & -0.230209000 & 3.449848000 \\ \mathrm{~F} & -3.070664000 & -0.304394000 & -1.798597000 \\ \mathrm{~F} & -1.192004000 & -0.837515000 & -2.779270000 \\ \mathrm{~F} & -1.030535000 & -3.025084000 & -1.031292000 \\ \mathrm{~F} & -3.080505000 & -2.616328000 & -0.488069000 \\ \mathrm{~F} & -2.561893000 & -2.942818000 & -2.541825000 \\ \mathrm{O} & 0.395034000 & 0.978394000 & 1.033878000 \\ \mathrm{O} & 2.423167000 & 1.553460000 & 1.824384000 \\ \mathrm{C} & -1.374193000 & 1.815559000 & -0.882262000 \\ \mathrm{C} & -0.614627000 & 3.100626000 & -0.520306000 \\ \mathrm{C} & -1.802619000 & -0.523857000 & 1.298946000 \\ \mathrm{C} & -1.705735000 & 0.192588000 & 2.656893000 \\ \mathrm{C} & -1.872344000 & -0.889475000 & -1.586990000 \\ \mathrm{C} & -2.160843000 & -2.389762000 & -1.405559000 \\ \mathrm{C} & 0.877427000 & -0.593767000 & -1.091340000 \\ \mathrm{H} & 0.741445000 & -1.632593000 & -1.414747000 \\ \mathrm{H} & 0.944375000 & 0.017600000 & -2.006157000 \\ \mathrm{C} & 3.751302000 & 0.363761000 & -1.056286000 \\ \mathrm{C} & 3.320454000 & 1.777377000 & -1.473543000 \\ \mathrm{H} & 2.405715000 & 1.774950000 & -2.082477000 \\ \mathrm{H} & 4.125950000 & 2.211414000 & -2.084827000 \\ \mathrm{H} & 3.158621000 & 2.427788000 & -0.603186000 \\ \mathrm{C} & 4.013534000 & -0.481804000 & -2.309028000 \\ \mathrm{H} & 4.325900000 & -1.506851000 & -2.071078000 \\ \mathrm{H} & 4.825598000 & -0.007134000 & -2.880649000 \\ \mathrm{H} & 3.129233000 & -0.526740000 & -2.961181000 \\ \mathrm{C} & 4.999750000 & 0.450234000 & -0.171817000 \\ \mathrm{H} & 4.779763000 & 0.951250000 & 0.782100000 \\ \mathrm{H} & 5.759843000 & 1.041075000 & -0.705124000 \\ \mathrm{H} & 5.429743000 & -0.541646000 & 0.025087000\end{array}$




$\begin{array}{ll}\mathrm{C} & 2.685376000 \\ \mathrm{C} & 3.318381000 \\ \mathrm{H} & 2.725025000 \\ \mathrm{H} & 3.368240000 \\ \mathrm{H} & 4.343911000 \\ \mathrm{C} & 3.587112000 \\ \mathrm{H} & 4.607355000 \\ \mathrm{H} & 3.649014000 \\ \mathrm{H} & 3.174378000 \\ \mathrm{C} & 1.323000000 \\ \mathrm{H} & 0.836869000 \\ \mathrm{H} & 1.490695000 \\ \mathrm{H} & 0.628154000 \\ \mathrm{C} & 1.675372000\end{array}$

$$
\begin{array}{r}
-1.882088000 \\
-2.971936000 \\
-3.184387000 \\
-3.900015000 \\
-2.714172000 \\
-1.545583000 \\
-1.276621000 \\
-2.443612000 \\
-0.728481000 \\
-2.351678000 \\
-1.587451000 \\
-3.231155000 \\
-2.653061000 \\
0.936487000
\end{array}
$$

0.921071000

0.050042000

\begin{tabular}{|c|c|c|c|}
\hline $\mathrm{Ge}$ & 0.638320000 & 0.130803000 & -0.004464000 \\
\hline $\mathrm{P}$ & -2.509808000 & 0.015433000 & 0.116492000 \\
\hline $\mathrm{F}$ & 1.306649000 & 2.587862000 & 1.144372000 \\
\hline $\mathrm{F}$ & -0.014829000 & 2.867982000 & -0.558994000 \\
\hline $\mathrm{F}$ & 2.658794000 & 3.707710000 & -0.731597000 \\
\hline $\mathrm{F}$ & 1.867019000 & 2.424433000 & -2.267191000 \\
\hline $\mathrm{F}$ & 3.210165000 & 1.622889000 & -0.785066000 \\
\hline $\mathrm{F}$ & 1.826942000 & -0.293187000 & -2.526993000 \\
\hline $\mathrm{F}$ & 2.974829000 & -1.105668000 & -0.867721000 \\
\hline $\mathrm{F}$ & 2.023829000 & -3.084256000 & -2.277052000 \\
\hline $\mathrm{F}$ & 0.851325000 & -2.922093000 & -0.472344000 \\
\hline $\mathrm{F}$ & 0.046017000 & -2.236071000 & -2.347497000 \\
\hline $\mathrm{F}$ & 2.963085000 & 0.480483000 & 1.676117000 \\
\hline $\mathrm{F}$ & 1.033972000 & 0.445995000 & 2.721510000 \\
\hline $\mathrm{F}$ & 2.831324000 & -2.241365000 & 1.437605000 \\
\hline $\mathrm{F}$ & 2.597823000 & -1.523051000 & 3.448597000 \\
\hline $\mathrm{F}$ & 0.882135000 & -2.227814000 & 2.353658000 \\
\hline O & -0.627147000 & 0.528963000 & -1.669650000 \\
\hline O & -2.689130000 & 1.205231000 & -2.296706000 \\
\hline $\mathrm{C}$ & 1.024247000 & 2.134366000 & -0.095821000 \\
\hline C & 2.220584000 & 2.486659000 & -0.997043000 \\
\hline C & 1.725743000 & -0.927513000 & -1.347244000 \\
\hline $\mathrm{C}$ & 1.161117000 & -2.323538000 & -1.632823000 \\
\hline C & 1.770693000 & -0.145653000 & 1.722093000 \\
\hline $\mathrm{C}$ & 2.039085000 & -1.559974000 & 2.247389000 \\
\hline C & -1.007332000 & -0.659171000 & 0.828530000 \\
\hline $\mathrm{H}$ & -0.945669000 & -1.734447000 & 0.603278000 \\
\hline $\mathrm{H}$ & -0.959465000 & -0.546540000 & 1.918202000 \\
\hline C & -3.671366000 & -1.365577000 & -0.353809000 \\
\hline C & -2.984355000 & -2.133749000 & -1.493474000 \\
\hline $\mathrm{H}$ & -2.836613000 & -1.504759000 & -2.382592000 \\
\hline $\mathrm{H}$ & -3.633686000 & -2.975829000 & -1.777436000 \\
\hline $\mathrm{H}$ & -2.014005000 & -2.552978000 & -1.193307000 \\
\hline $\mathrm{C}$ & -3.898879000 & -2.318156000 & 0.825967000 \\
\hline $\mathrm{H}$ & -2.955880000 & -2.749840000 & 1.192467000 \\
\hline $\mathrm{H}$ & -4.530457000 & -3.150703000 & 0.479914000 \\
\hline $\mathrm{H}$ & -4.412680000 & -1.837537000 & 1.667192000 \\
\hline C & -4.995315000 & -0.780872000 & -0.859345000 \\
\hline $\mathrm{H}$ & -5.581689000 & -0.331134000 & -0.045702000 \\
\hline $\mathrm{H}$ & -5.594485000 & -1.599151000 & -1.287153000 \\
\hline $\mathrm{H}$ & -4.825141000 & -0.028789000 & -1.643394000 \\
\hline C & -3.204680000 & 1.341023000 & 1.226835000 \\
\hline $\mathrm{C}$ & -3.957790000 & 0.716848000 & 2.406427000 \\
\hline $\mathrm{H}$ & -4.875816000 & 0.205769000 & 2.086323000 \\
\hline $\mathrm{H}$ & -4.250972000 & 1.525004000 & 3.09381500 \\
\hline
\end{tabular}

$-0.852222000$

0.639597000

$-0.249795000$

2.118202000

1.821216000

2.751323000

2.725444000

1. 451660000

2.074882000

2.091496000

0.656102000

1.116165000
3-Ge $\left(\mathrm{CO}_{2}\right): E=-4684.968490$ 


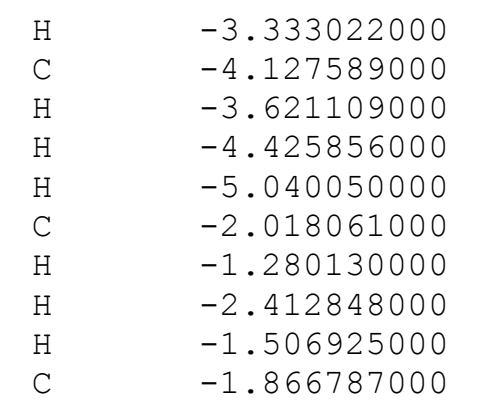

0.009302000

2.262844000

2. 668285000

3.100677000

1.753046000

2.164420000

1.568357000

2. 924807000

2.693437000

0.730981000

3-Sn $\left(\mathrm{CO}_{2}\right): E=-2822.435788$

\begin{tabular}{|c|c|c|c|}
\hline $\mathrm{Sn}$ & 0.647414000 & 0.133019000 & -0.042128000 \\
\hline $\mathrm{P}$ & -2.645790000 & 0.166159000 & 0.132817000 \\
\hline $\mathrm{F}$ & 1.730002000 & 2.668860000 & 1.001706000 \\
\hline $\mathrm{F}$ & 0.586310000 & 3.104006000 & -0.802127000 \\
\hline $\mathrm{F}$ & 3.407355000 & 3.325786000 & -0.894686000 \\
\hline $\mathrm{F}$ & 2.413287000 & 2.082506000 & -2.348898000 \\
\hline $\mathrm{F}$ & 3.464367000 & 1.175700000 & -0.701489000 \\
\hline $\mathrm{F}$ & 1.616868000 & -0.722408000 & -2.739438000 \\
\hline $\mathrm{F}$ & 2.774105000 & -1.642143000 & -1.134744000 \\
\hline $\mathrm{F}$ & 1.235208000 & -3.435468000 & -2.431001000 \\
\hline $\mathrm{F}$ & 0.555428000 & -3.081926000 & -0.412924000 \\
\hline $\mathrm{F}$ & -0.521841000 & -2.234769000 & -2.073868000 \\
\hline $\mathrm{F}$ & 3.013321000 & 0.338647000 & 1.924166000 \\
\hline $\mathrm{F}$ & 1.028335000 & 0.423784000 & 2.853113000 \\
\hline $\mathrm{F}$ & 2.667467000 & -2.343656000 & 1.519362000 \\
\hline $\mathrm{F}$ & 2.473752000 & -1.727566000 & 3.575156000 \\
\hline $\mathrm{F}$ & 0.717239000 & -2.224644000 & 2.429259000 \\
\hline O & -0.786536000 & 0.753162000 & -1.688519000 \\
\hline O & -2.913566000 & 0.977632000 & -2.407442000 \\
\hline $\mathrm{C}$ & 1.423217000 & 2.212427000 & -0.234814000 \\
\hline $\mathrm{C}$ & 2.709708000 & 2.216535000 & -1.066460000 \\
\hline C & 1.534127000 & -1.266434000 & -1.512301000 \\
\hline $\mathrm{C}$ & 0.697986000 & -2.538841000 & -1.628375000 \\
\hline $\mathrm{C}$ & 1.782202000 & -0.209415000 & 1.894653000 \\
\hline C & 1.925627000 & -1.650213000 & 2.371300000 \\
\hline C & -1.216508000 & -0.595897000 & 0.888256000 \\
\hline $\mathrm{H}$ & -1.222779000 & -1.681198000 & 0.709583000 \\
\hline $\mathrm{H}$ & -1.159090000 & -0.428192000 & 1.971691000 \\
\hline $\mathrm{C}$ & -4.028317000 & -1.062532000 & -0.083070000 \\
\hline $\mathrm{C}$ & -3.558878000 & -2.044568000 & -1.167731000 \\
\hline $\mathrm{H}$ & -3.414261000 & -1.539734000 & -2.132703000 \\
\hline $\mathrm{H}$ & -4.336397000 & -2.813243000 & -1.294086000 \\
\hline $\mathrm{H}$ & -2.626580000 & -2.558187000 & -0.893617000 \\
\hline C & -4.271415000 & -1.824335000 & 1.224888000 \\
\hline $\mathrm{H}$ & -3.369549000 & -2.354709000 & 1.563043000 \\
\hline $\mathrm{H}$ & -5.053809000 & -2.578080000 & 1.046618000 \\
\hline $\mathrm{H}$ & -4.616518000 & -1.170375000 & 2.035760000 \\
\hline $\mathrm{C}$ & -5.304537000 & -0.356210000 & -0.554473000 \\
\hline $\mathrm{H}$ & -5.750546000 & 0.256907000 & 0.241572000 \\
\hline $\mathrm{H}$ & -6.041925000 & -1.125214000 & -0.831663000 \\
\hline $\mathrm{H}$ & -5.106981000 & 0.271154000 & -1.435037000 \\
\hline $\mathrm{C}$ & -3.045130000 & 1.724715000 & 1.076195000 \\
\hline C & -3.727447000 & 1.390910000 & 2.406768000 \\
\hline $\mathrm{H}$ & -4.742659000 & 0.996649000 & 2.260324000 \\
\hline $\mathrm{H}$ & -3.812107000 & 2.316311000 & 2.996862000 \\
\hline $\mathrm{H}$ & -3.148388000 & 0.668719000 & 3.002284000 \\
\hline $\mathrm{C}$ & -3.928386000 & 2.650594000 & 0.228449000 \\
\hline $\mathrm{H}$ & -3.461495000 & 2.891317000 & -0.736089000 \\
\hline $\mathrm{H}$ & -4.072814000 & 3.588303000 & 0.7863 \\
\hline
\end{tabular}
2.972440000
0.416491000
$-0.469328000$
1.065144000
0.084231000
1.750323000
2. 306277000
2.440998000
0.937853000
$-1.552751000$

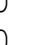

0




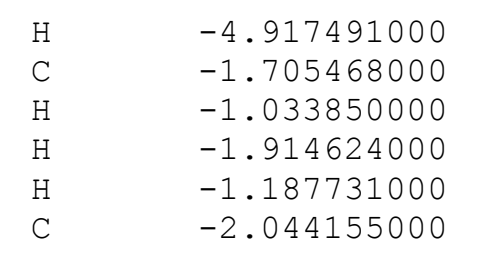
2.221364000
2. 432765000
1. 853522000
3. 382163000
2. 685296000
0.715025000

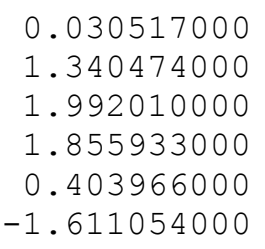

3-Sn' $\quad\left(\mathbf{C O}_{2}\right): E=-1335.257308$

\begin{tabular}{|c|c|c|c|}
\hline $\mathrm{Sn}$ & -1.850897000 & -0.013736000 & 0.059982000 \\
\hline $\mathrm{P}$ & 1.608542000 & -0.022041000 & 0.073155000 \\
\hline O & -0.070333000 & -0.508884000 & -1.916516000 \\
\hline O & 2.088264000 & -0.232930000 & -2.562569000 \\
\hline C & -2.380356000 & -2.053105000 & -0.440330000 \\
\hline $\mathrm{C}$ & -3.878082000 & -2.161081000 & -0.734971000 \\
\hline $\mathrm{C}$ & -2.326494000 & 1.466812000 & -1.447244000 \\
\hline C & -1.293515000 & 2.589953000 & -1.519774000 \\
\hline C & -3.047386000 & 0.511835000 & 1.826204000 \\
\hline C & -2.838138000 & 1.961180000 & 2.268596000 \\
\hline C & 0.137029000 & 0.494201000 & 0.961462000 \\
\hline $\mathrm{H}$ & 0.129928000 & 1.595911000 & 0.974489000 \\
\hline $\mathrm{H}$ & 0.161283000 & 0.153847000 & 2.008247000 \\
\hline C & 2.932777000 & 1.288114000 & 0.217953000 \\
\hline C & 2.450048000 & 2.462092000 & -0.650190000 \\
\hline $\mathrm{H}$ & 2.344612000 & 2.160171000 & -1.701779000 \\
\hline $\mathrm{H}$ & 3.194868000 & 3.270800000 & -0.591574000 \\
\hline $\mathrm{H}$ & 1.491184000 & 2.869961000 & -0.296794000 \\
\hline $\mathrm{C}$ & 3.094072000 & 1.764278000 & 1.665831000 \\
\hline $\mathrm{H}$ & 2.149895000 & 2.150189000 & 2.076840000 \\
\hline $\mathrm{H}$ & 3.826644000 & 2.586672000 & 1.684619000 \\
\hline $\mathrm{H}$ & 3.464759000 & 0.974200000 & 2.331809000 \\
\hline C & 4.267036000 & 0.777393000 & -0.337748000 \\
\hline $\mathrm{H}$ & 4.718039000 & 0.020391000 & 0.319378000 \\
\hline $\mathrm{H}$ & 4.968382000 & 1.624645000 & -0.394312000 \\
\hline $\mathrm{H}$ & 4.136287000 & 0.359575000 & -1.345875000 \\
\hline $\mathrm{C}$ & 2.115162000 & -1.715892000 & 0.682618000 \\
\hline $\mathrm{C}$ & 2.768424000 & -1.650001000 & 2.065700000 \\
\hline $\mathrm{H}$ & 3.759448000 & -1.176039000 & 2.026980000 \\
\hline $\mathrm{H}$ & 2.905463000 & -2.675442000 & 2.443669000 \\
\hline $\mathrm{H}$ & 2.146097000 & -1.106579000 & 2.792888000 \\
\hline C & 3.061974000 & -2.383450000 & -0.325684000 \\
\hline $\mathrm{H}$ & 2.609158000 & -2.458400000 & -1.323535000 \\
\hline $\mathrm{H}$ & 3.282457000 & -3.400716000 & 0.034262000 \\
\hline $\mathrm{H}$ & 4.014083000 & -1.849918000 & -0.429513000 \\
\hline $\mathrm{C}$ & 0.825793000 & -2.547525000 & 0.753101000 \\
\hline $\mathrm{H}$ & 0.141494000 & -2.185888000 & 1.534685000 \\
\hline $\mathrm{H}$ & 1.091584000 & -3.588183000 & 0.994953000 \\
\hline $\mathrm{H}$ & 0.294458000 & -2.543040000 & -0.209995000 \\
\hline C & 1.137506000 & -0.282791000 & -1.805443000 \\
\hline $\mathrm{H}$ & -1.780498000 & -2.332252000 & -1.317929000 \\
\hline $\mathrm{H}$ & -2.101860000 & -2.724407000 & 0.386207000 \\
\hline $\mathrm{H}$ & -4.487220000 & -1.873280000 & 0.135924000 \\
\hline $\mathrm{H}$ & -4.175312000 & -1.510423000 & -1.572208000 \\
\hline $\mathrm{H}$ & -4.166670000 & -3.187997000 & -1.007783000 \\
\hline $\mathrm{H}$ & -4.108764000 & 0.339408000 & 1.583129000 \\
\hline $\mathrm{H}$ & -2.807499000 & -0.182963000 & 2.648151000 \\
\hline $\mathrm{H}$ & -1.790626000 & 2.152002000 & 2.553693000 \\
\hline $\mathrm{H}$ & -3.085170000 & 2.669289000 & 1.461076000 \\
\hline $\mathrm{H}$ & -3.459147000 & 2.234111000 & 3.136813000 \\
\hline $\mathrm{H}$ & -2.367678000 & 0.933599000 & -2.407678000 \\
\hline $\mathrm{H}$ & -3.331524000 & 1.868473000 & -1.243502000 \\
\hline $\mathrm{H}$ & -1.534530000 & 3.323708000 & -2.304460000 \\
\hline
\end{tabular}


3-B $\left(\mathrm{CO}_{2}\right): E=-1371.320067$

\begin{tabular}{|c|c|c|c|}
\hline C & -0.954408000 & 0.041478000 & 1.564931000 \\
\hline 0 & 0.326519000 & 0.036526000 & 1.472838000 \\
\hline $\mathrm{C}$ & -0.153788000 & 0.058621000 & -1.097609000 \\
\hline $\mathrm{H}$ & -0.105374000 & 0.983354000 & -1.688108000 \\
\hline $\mathrm{H}$ & -0.087973000 & -0.797719000 & -1.782233000 \\
\hline 0 & -1.639631000 & 0.070610000 & 2.554803000 \\
\hline B & 1.052890000 & 0.024542000 & 0.106980000 \\
\hline $\mathrm{P}$ & -1.676418000 & -0.000299000 & -0.178100000 \\
\hline C & -2.658789000 & 1.566563000 & -0.354957000 \\
\hline C & -3.915906000 & 1.531344000 & 0.521603000 \\
\hline $\mathrm{H}$ & -3.684961000 & 1.195714000 & 1.543151000 \\
\hline $\mathrm{H}$ & -4.327762000 & 2.550693000 & 0.577766000 \\
\hline $\mathrm{H}$ & -4.693096000 & 0.883581000 & 0.094115000 \\
\hline C & -1.718447000 & 2.679330000 & 0.143036000 \\
\hline $\mathrm{H}$ & -0.751262000 & 2.691589000 & -0.382204000 \\
\hline $\mathrm{H}$ & -2.209425000 & 3.649302000 & -0.028697000 \\
\hline $\mathrm{H}$ & -1.522060000 & 2.585273000 & 1.221186000 \\
\hline $\mathrm{C}$ & -3.019267000 & 1.803955000 & -1.825089000 \\
\hline $\mathrm{H}$ & -3.691409000 & 1.028825000 & -2.218207000 \\
\hline $\mathrm{H}$ & -3.540187000 & 2.770468000 & -1.906094000 \\
\hline $\mathrm{H}$ & -2.125979000 & 1.850627000 & -2.464367000 \\
\hline $\mathrm{C}$ & -2.529595000 & -1.645220000 & -0.325048000 \\
\hline C & -3.076764000 & -1.832791000 & -1.742985000 \\
\hline $\mathrm{H}$ & -3.463610000 & -2.858922000 & -1.839569000 \\
\hline $\mathrm{H}$ & -3.906734000 & -1.143706000 & -1.954068000 \\
\hline $\mathrm{H}$ & -2.297575000 & -1.694156000 & -2.507284000 \\
\hline $\mathrm{C}$ & -1.436851000 & -2.690015000 & -0.032787000 \\
\hline $\mathrm{H}$ & -1.021706000 & -2.580258000 & 0.980767000 \\
\hline $\mathrm{H}$ & -1.892683000 & -3.689193000 & -0.104239000 \\
\hline $\mathrm{H}$ & -0.598124000 & -2.647349000 & -0.742188000 \\
\hline C & -3.646874000 & -1.783369000 & 0.717174000 \\
\hline $\mathrm{H}$ & -4.518441000 & -1.164263000 & 0.472756000 \\
\hline $\mathrm{H}$ & -3.977312000 & -2.833440000 & 0.726293000 \\
\hline $\mathrm{H}$ & -3.297546000 & -1.520999000 & 1.725490000 \\
\hline C & 1.927778000 & 1.381720000 & 0.018020000 \\
\hline $\mathrm{C}$ & 2.652203000 & 1.696012000 & -1.143935000 \\
\hline C & 1.969013000 & 2.312262000 & 1.066068000 \\
\hline C & 3.374920000 & 2.881775000 & -1.261430000 \\
\hline $\mathrm{H}$ & 2.657947000 & 0.992211000 & -1.982397000 \\
\hline C & 2.688957000 & 3.504894000 & 0.959310000 \\
\hline $\mathrm{H}$ & 1.428714000 & 2.095269000 & 1.990623000 \\
\hline $\mathrm{C}$ & 3.393117000 & 3.796027000 & -0.206763000 \\
\hline $\mathrm{H}$ & 3.929663000 & 3.094889000 & -2.177582000 \\
\hline $\mathrm{H}$ & 2.701805000 & 4.208235000 & 1.794614000 \\
\hline $\mathrm{H}$ & 3.957488000 & 4.726328000 & -0.293278000 \\
\hline C & 1.901131000 & -1.348751000 & 0.028772000 \\
\hline $\mathrm{C}$ & 2.240768000 & -2.053132000 & 1.194694000 \\
\hline $\mathrm{C}$ & 2.337097000 & -1.890382000 & -1.190897000 \\
\hline $\mathrm{C}$ & 2.969745000 & -3.241913000 & 1.146598000 \\
\hline $\mathrm{H}$ & 1.918456000 & -1.659293000 & 2.161592000 \\
\hline $\mathrm{C}$ & 3.072549000 & -3.074845000 & -1.251645000 \\
\hline $\mathrm{H}$ & 2.097186000 & -1.378046000 & -2.128639000 \\
\hline $\mathrm{C}$ & 3.389119000 & -3.758989000 & -0.078597000 \\
\hline $\mathrm{H}$ & 3.214099000 & -3.767944000 & 2.071872000 \\
\hline $\mathrm{H}$ & 3.399097000 & -3.466176000 & -2.217465000 \\
\hline $\mathrm{H}$ & 3.961230000 & -4.687655000 & -0.118906000 \\
\hline
\end{tabular}




\section{Reaction with PhNCO}

\begin{tabular}{|c|c|c|c|}
\hline $\mathrm{Si}$ & & & \\
\hline$\Delta \perp$ & $-1.6 / 43 / 5000$ & -0.084458000 & -0.038845000 \\
\hline $\mathrm{C}$ & -0.490557000 & 0.014531000 & 1.380413000 \\
\hline $\mathrm{H}$ & -0.908170000 & 0.732602000 & 2.105452000 \\
\hline $\mathrm{H}$ & -0.597810000 & -0.972581000 & 1.852896000 \\
\hline $\mathrm{C}$ & -2.252149000 & 1.579544000 & -0.849112000 \\
\hline $\mathrm{C}$ & -3.179056000 & 2.461346000 & 0.005536000 \\
\hline $\mathrm{C}$ & -3.323651000 & -0.880180000 & 0.621066000 \\
\hline $\mathrm{C}$ & -3.300521000 & -2.363635000 & 1.019194000 \\
\hline C & -1.200958000 & -1.263865000 & -1.506025000 \\
\hline $\mathrm{C}$ & -0.214823000 & -0.813280000 & -2.590044000 \\
\hline $\mathrm{P}$ & 1.348406000 & 0.302088000 & 1.069819000 \\
\hline $\mathrm{C}$ & 2.043100000 & -1.156951000 & 2.079213000 \\
\hline $\mathrm{C}$ & 1.625056000 & 1.934541000 & 1.997953000 \\
\hline $\mathrm{C}$ & 1.412619000 & -1.451948000 & 3.444042000 \\
\hline $\mathrm{H}$ & 0.341226000 & -1.691965000 & 3.381650000 \\
\hline $\mathrm{H}$ & 1.905967000 & -2.340578000 & 3.871317000 \\
\hline $\mathrm{H}$ & 1.539425000 & -0.631264000 & 4.159307000 \\
\hline $\mathrm{C}$ & 1.818100000 & -2.375552000 & 1.170190000 \\
\hline $\mathrm{H}$ & 0.748159000 & -2.581912000 & 1.011675000 \\
\hline $\mathrm{H}$ & 2.285738000 & -2.233193000 & 0.185316000 \\
\hline $\mathrm{H}$ & 2.260555000 & -3.269416000 & 1.639078000 \\
\hline $\mathrm{C}$ & 3.549998000 & -0.944136000 & 2.263215000 \\
\hline $\mathrm{H}$ & 4.018687000 & -1.893240000 & 2.570625000 \\
\hline $\mathrm{H}$ & 4.038540000 & -0.614914000 & 1.333672000 \\
\hline $\mathrm{H}$ & 3.757672000 & -0.204365000 & 3.050249000 \\
\hline $\mathrm{C}$ & 1.356603000 & 1.910970000 & 3.501672000 \\
\hline $\mathrm{H}$ & 2.127801000 & 1.344265000 & 4.041906000 \\
\hline $\mathrm{H}$ & 1.372620000 & 2.941911000 & 3.891949000 \\
\hline $\mathrm{H}$ & 0.372819000 & 1.481574000 & 3.747053000 \\
\hline $\mathrm{C}$ & 3.070258000 & 2.384675000 & 1.738107000 \\
\hline $\mathrm{H}$ & 3.272835000 & 2.468814000 & 0.660490000 \\
\hline $\mathrm{H}$ & 3.224494000 & 3.378039000 & 2.190310000 \\
\hline $\mathrm{H}$ & 3.811715000 & 1.700660000 & 2.170977000 \\
\hline $\mathrm{C}$ & 0.695801000 & 2.953347000 & 1.322386000 \\
\hline $\mathrm{H}$ & -0.364085000 & 2.767886000 & 1.545249000 \\
\hline $\mathrm{H}$ & 0.934458000 & 3.961336000 & 1.698127000 \\
\hline $\mathrm{H}$ & 0.821383000 & 2.958144000 & 0.229181000 \\
\hline F & -0.580330000 & 0.354878000 & -3.084794000 \\
\hline $\mathrm{F}$ & -0.174887000 & -1.704895000 & -3.566809000 \\
\hline F & 1.008514000 & -0.704598000 & -2.074869000 \\
\hline F & -0.698544000 & -2.409325000 & -0.976866000 \\
\hline F & -2.351028000 & -1.567231000 & -2.149254000 \\
\hline F & -1.159293000 & 2.319926000 & -1.132632000 \\
\hline F & -2.904666000 & 1.324870000 & -2.002538000 \\
\hline F & -4.392806000 & 1.943009000 & 0.054086000 \\
\hline F & -2.699886000 & 2.528690000 & 1.252467000 \\
\hline F & -3.248908000 & 3.684802000 & -0.484710000 \\
\hline F & -3.181978000 & -3.129295000 & -0.050721000 \\
\hline F & -2.260875000 & -2.601030000 & 1.825261000 \\
\hline F & -4.409184000 & -2.687150000 & 1.660881000 \\
\hline F & -3.644015000 & -0.192742000 & 1.750853000 \\
\hline F & -4.332081000 & -0.734835000 & -0.260200000 \\
\hline $\mathrm{C}$ & 5.657665000 & 0.721352000 & -0.546501000 \\
\hline $\mathrm{C}$ & 4.490890000 & 0.360216000 & -1.229585000 \\
\hline $\mathrm{C}$ & 6.526194000 & -0.267297000 & -0.090624000 \\
\hline $\mathrm{H}$ & 7.431440000 & 0.019252000 & 0.446647000 \\
\hline C & 4.207403000 & -0.991143000 & -1.466405000 \\
\hline
\end{tabular}




$\begin{array}{ll}\mathrm{C} & 6.244557000 \\ \mathrm{H} & 3.299312000 \\ \mathrm{H} & 6.928089000 \\ \mathrm{C} & 5.085334000 \\ \mathrm{H} & 4.858210000 \\ \mathrm{H} & 5.864651000 \\ \mathrm{~N} & 3.633856000 \\ \mathrm{C} & 2.508645000 \\ \mathrm{O} & 1.464887000\end{array}$

RC-1-Ge (PhNCO) : $\mathrm{E}=-4895.781462$

\section{$\mathrm{Ge}$}

C

$\mathrm{H}$

$\mathrm{H}$

C

C

C

C

C

C
$-1.614477000$

$-1.258577000$

$-2.384998000$

$-1.969672000$

$-3.021059000$

1.778722000

1.358894000

1.704529000

2.160066000
-0.317417000
-2.006617000
0.040787000
-1.007978000
-1.192109000
-0.379398000
-1.670714000
-1.893983000
-2.145941000

$-0.074403000$

$-0.897645000$

$-1.711262000$

$-1.377459000$

1. 823172000

2.968240000

$-0.729180000$

$-1.310231000$

$-1.034946000$

$-0.850478000$

$-0.016845000$

$-1.427560000$

0.083039000

$-2.857005000$

$-3.115344000$

$-3.562990000$

$-3.033473000$

$-1.263090000$

$-1.350520000$

$-0.287165000$

$-2.051920000$

$-1.195143000$

$-1.844882000$

$-0.152760000$

$-1.441198000$

$-1.242440000$

$-1.809880000$

$-1.042608000$

$-1.878640000$

0.921132000

1.888428000

1.120669000

0.412506000

0.884163000

0.313675000

1.136426000

1. 827115000

0.388933000

$-1.662292000$

$-1.168788000$

$-2.359909000$

$-0.775398000$

2.167137000

1. 780155000

4.131593000

2.812468000

2.985067000

$-0.431152000$

$-2.396473000$

$-1.647913000$ 


$\begin{array}{ll}F & 2.951659000 \\ F & 3.769510000 \\ C & -5.821860000 \\ C & -4.727431000 \\ C & -7.105513000 \\ \mathrm{H} & -7.960183000 \\ \mathrm{C} & -4.914195000 \\ \mathrm{C} & -7.300479000 \\ \mathrm{H} & -4.046469000 \\ \mathrm{H} & -8.307818000 \\ \mathrm{C} & -6.202887000 \\ \mathrm{H} & -6.349225000 \\ \mathrm{H} & -5.649569000 \\ \mathrm{~N} & -3.448279000 \\ \mathrm{C} & -2.263860000 \\ \mathrm{O} & -1.090461000\end{array}$

RC-1-Sn (PhNCO) : $E=-3033.243455$

$\begin{array}{lrr}\mathrm{Sn} & 1.218313000 & -0.229183000 \\ \mathrm{C} & -0.262453000 & 1.013370000 \\ \mathrm{H} & 0.303317000 & 1.601803000 \\ \mathrm{H} & -0.868828000 & 0.281208000 \\ \mathrm{C} & 2.018948000 & -0.876899000 \\ \mathrm{C} & 2.090039000 & 0.114493000 \\ \mathrm{C} & 3.090695000 & 0.697767000 \\ \mathrm{C} & 3.755584000 & -0.065406000 \\ \mathrm{C} & 1.080029000 & -2.251450000 \\ \mathrm{C} & -0.235388000 & -3.011950000 \\ \mathrm{P} & -1.451489000 & 2.059910000 \\ \mathrm{C} & -2.658753000 & 2.509990000 \\ \mathrm{C} & -0.373386000 & 3.559947000 \\ \mathrm{C} & -2.033205000 & 2.711891000 \\ \mathrm{H} & -1.571523000 & 1.789402000 \\ \mathrm{H} & -2.821458000 & 2.998122000 \\ \mathrm{H} & -1.273104000 & 3.503659000 \\ \mathrm{C} & -3.626887000 & 1.318295000 \\ \mathrm{H} & -3.104068000 & 0.364742000 \\ \mathrm{H} & -4.190782000 & 1.215646000 \\ \mathrm{H} & -4.349108000 & 1.461203000 \\ \mathrm{C} & -3.461813000 & 3.755356000 \\ \mathrm{H} & -4.308427000 & 3.874385000 \\ \mathrm{H} & -3.872749000 & 3.671581000 \\ \mathrm{H} & -2.855282000 & 4.670295000 \\ \mathrm{C} & 0.138349000 & 4.398072000 \\ \mathrm{H} & -0.674318000 & 4.928117000 \\ \mathrm{H} & 0.841269000 & 5.159175000 \\ \mathrm{H} & 0.686431000 & 3.788675000 \\ \mathrm{C} & -1.166516000 & 4.440545000 \\ \mathrm{H} & -1.491495000 & 3.860334000 \\ \mathrm{H} & -0.524575000 & 5.263413000 \\ \mathrm{H} & -2.055383000 & 4.887530000 \\ \mathrm{C} & 0.828414000 & 2.989774000 \\ \mathrm{H} & 1.569545000 & 2.537882000 \\ \mathrm{H} & 1.355025000 & 3.799254000 \\ \mathrm{H} & 0.510964000 & 2.258432000 \\ \mathrm{~F} & -0.397522000 & -3.390503000 \\ \mathrm{~F} & -0.290455000 & -4.079952000 \\ \mathrm{~F} & -1.265264000 & -2.210040000 \\ \mathrm{~F} & 1.227229000 & -2.087812000 \\ \mathrm{~F} & 1.361072000 & -3.069416000 \\ \mathrm{~F} & & -1.963900000 \\ \mathrm{H} & -35571000 & \end{array}$

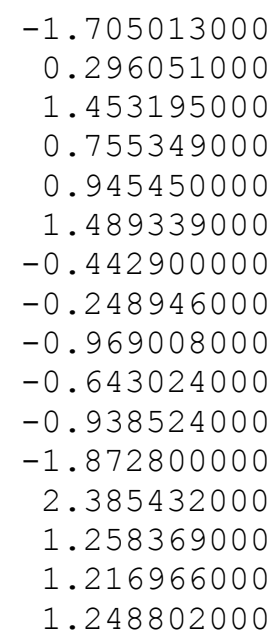

$-0.041378000$

$-0.971730000$

$-1.711470000$

$-1.528812000$

1.929752000

3.086581000

$-0.880804000$

$-2.022412000$

$-0.977441000$

$-0.795761000$

0.002722000

$-1.401113000$

0.430009000

$-2.784032000$

$-3.163125000$

$-3.499874000$

$-2.791769000$

$-1.461363000$

$-1.645953000$

$-0.522542000$

$-2.281943000$

$-1.010880000$

$-1.706302000$

0.007372000

$-1.068081000$

$-0.741271000$

$-1.257439000$

$-0.363692000$

$-1.475619000$

1.406270000

2.282745000

1. 759776000

0.943064000

1.197594000

0.520804000

1. 727753000

1.957656000

0.460314000

$-1.573808000$

$-1.126329000$

$-2.318541000$

$-0.546394000$

2.366539000 


$\begin{array}{ll}\text { F } & 3.291068000 \\ F & 2.704056000 \\ F & 2.756143000 \\ F & 0.864965000 \\ F & 3.973890000 \\ F & 2.946852000 \\ F & 4.909765000 \\ F & 2.777427000 \\ F & 4.017499000 \\ C & -5.583118000 \\ C & -4.535960000 \\ C & -6.888486000 \\ H & -7.707296000 \\ C & -4.788455000 \\ C & -7.149655000 \\ H & -3.953225000 \\ H & -8.173700000 \\ C & -6.098381000 \\ H & -6.299094000 \\ H & -5.357310000 \\ \text { N } & -3.233511000 \\ \mathrm{C} & -2.065943000 \\ \mathrm{O} & -0.897896000 \\ & \end{array}$

$$
\begin{array}{r}
-1.255605000 \\
-0.412702000 \\
1.201335000 \\
0.478445000 \\
-1.323054000 \\
-0.066894000 \\
0.479474000 \\
1.928085000 \\
0.886174000 \\
-0.605591000 \\
-1.202618000 \\
-0.752676000 \\
-0.284510000 \\
-1.949883000 \\
-1.488790000 \\
-2.406027000 \\
-1.598395000 \\
-2.086208000 \\
-2.664408000 \\
-0.028657000 \\
-1.035042000 \\
-0.935721000 \\
-0.855455000
\end{array}
$$

1. 660205000

4.133448000

2. 706037000

3.455710000

$-1.653260000$

$-3.078849000$

$-2.374756000$

$-1.381772000$

0.081403000

1.447553000

0.739851000

0.986522000

1.534403000

$-0.416176000$

$-0.168894000$

$-0.948490000$

$-0.527335000$

$-0.865078000$

$-1.767938000$

2. 344283000

1.176449000

1. 289580000

1. 448177000

1.234501000

2. 182601000

1. 374308000

$-2.154239000$

$-1.924115000$

0.010433000

1. 141861000

0.265049000

$-0.463186000$

0.899387000

$-0.287616000$

2.113020000

2. 762604000

1.600474000

2.767926000

1. 972313000

1.335480000

2. 661226000

2.589517000

$-1.188975000$

$-1.636275000$

$-0.847985000$

$-1.931038000$

$-2.508271000$

$-0.645855000$

$-1.396204000$

$-0.444938000$

0.275955000

$-2.418617000$

$-2.180505000$

$-3.174945000$

$-2.869575000$

$-1.771653000$

0.871830000

1.072079000

1.015321000 


$\begin{array}{lr}\mathrm{C} & -1.065128000 \\ \mathrm{C} & -0.792817000 \\ \mathrm{H} & 1.285467000 \\ \mathrm{C} & -2.175390000 \\ \mathrm{H} & -1.200494000 \\ \mathrm{C} & -2.040679000 \\ \mathrm{H} & -0.679471000 \\ \mathrm{H} & -3.149898000 \\ \mathrm{H} & -2.908016000 \\ \mathrm{C} & 2.841705000 \\ \mathrm{C} & 4.019056000 \\ \mathrm{C} & 2.961255000 \\ \mathrm{C} & 5.259752000 \\ \mathrm{H} & 3.965096000 \\ \mathrm{C} & 4.203057000 \\ \mathrm{H} & 2.067595000 \\ \mathrm{C} & 5.354630000 \\ \mathrm{H} & 6.156278000 \\ \mathrm{H} & 4.272379000 \\ \mathrm{H} & 6.325928000 \\ \mathrm{C} & -3.294780000 \\ \mathrm{C} & -3.369274000 \\ \mathrm{C} & -4.402462000 \\ \mathrm{C} & -4.550446000 \\ \mathrm{C} & -5.578594000 \\ \mathrm{C} & -5.656922000 \\ \mathrm{H} & -2.483820000 \\ \mathrm{H} & -4.318193000 \\ \mathrm{H} & -4.606407000 \\ \mathrm{H} & -6.440398000 \\ \mathrm{H} & -6.579183000 \\ & \\ \mathrm{H} & \\ \mathrm{H} & -140\end{array}$

$$
\begin{array}{r}
-1.186126000 \\
-3.946104000 \\
-3.577168000 \\
-2.000425000 \\
-0.104247000 \\
-3.386428000 \\
-5.030424000 \\
-1.550860000 \\
-4.030550000 \\
-1.397720000 \\
-1.038387000 \\
-2.283149000 \\
-1.555573000 \\
-0.344230000 \\
-2.778264000 \\
-2.558305000 \\
-2.422844000 \\
-1.274122000 \\
-3.447364000 \\
-2.817739000 \\
-0.847293000 \\
0.452726000 \\
-1.697136000 \\
0.886273000 \\
-1.250082000 \\
0.038698000 \\
1.090501000 \\
-2.705301000 \\
1.897959000 \\
-1.916844000 \\
0.384415000
\end{array}
$$

TS-1-Si (PhNCO) : $E=-3108.403542$

$\mathrm{Si}$

C

$\mathrm{H}$

$\mathrm{H}$

C

C

C

C

C

C
1.379498000

0.799882000

1. 601616000

0.909105000

1. 435130000

1. 990401000

3.320867000

3.798605000

0.856235000

$-0.555262000$

$-0.855641000$

$-1.160258000$

$-0.506813000$

0.032214000

0.895672000

$-0.260551000$

0.360197000

$-1.573491000$

$-0.773954000$

$-2.463337000$

$-1.804561000$

$-2.352647000$

$-2.700623000$

$-3.192618000$

$-2.080777000$

0.395702000

$-0.101855000$

0.647076000
$-0.414390000$

1. 342019000

1. 998137000

1. 348029000

$-1.650084000$

$-1.074555000$

$-0.115980000$

0.116961000

$-1.535582000$

$-2.127760000$

2. 080701000

3. 123972000

3. 109872000

3.937052000

3. 302354000

4. 459927000

4.696409000

2. 095510000

1. 369434000

1.531223000

2. 619505000

4.045040000

4.486761000

3.488700000

4. 872402000

4. 325036000

5.106832000

4.761859000
1. 363031000

1. 218176000

0.822933000

1.571393000

1.409072000

1.492870000

1.169113000

1.775478000

1.650637000

0.423687000

1.102572000

$-0.662363000$

0.733665000

1. 945721000

$-1.056805000$

$-1.226646000$

$-0.354116000$

1. 289040000

$-1.916277000$

$-0.657441000$

$-1.213720000$

$-0.698769000$

$-1.147771000$

$-0.103548000$

$-0.550772000$

$-0.023294000$

$-0.752899000$

$-1.553723000$

0.303076000

$-0.495973000$

0.445256000

0.026006000

$-0.277528000$

0.092304000

$-1.375522000$

1. 564339000

2.882289000

$-0.272163000$

$-1.715661000$

$-1.483871000$

$-1.591183000$

0.084889000

$-1.453841000$

1.623428000

$-1.962412000$

$-2.210439000$

$-2.887557000$

$-1.241764000$

$-2.520554000$

$-2.735585000$

$-2.205003000$

$-3.461616000$

$-1.174210000$

$-2.121448000$

$-0.729308000$

$-0.502883000$

1. 415208000

0.823969000

2.395616000 


$\begin{array}{lr}\mathrm{H} & 1.343347000 \\ \mathrm{C} & -1.856438000 \\ \mathrm{H} & -2.513227000 \\ \mathrm{H} & -1.686519000 \\ \mathrm{H} & -2.387801000 \\ \mathrm{C} & 0.154811000 \\ \mathrm{H} & 1.191052000 \\ \mathrm{H} & 0.193925000 \\ \mathrm{H} & -0.410807000 \\ \mathrm{~F} & -0.834900000 \\ \mathrm{~F} & -0.637059000 \\ \mathrm{~F} & -1.455016000 \\ \mathrm{~F} & 1.011774000 \\ \mathrm{~F} & 1.706276000 \\ \mathrm{~F} & 0.299478000 \\ \mathrm{~F} & 2.309937000 \\ \mathrm{~F} & 2.593148000 \\ \mathrm{~F} & 2.868967000 \\ \mathrm{~F} & 1.010227000 \\ \mathrm{~F} & 3.675191000 \\ \mathrm{~F} & 3.082289000 \\ \mathrm{~F} & 5.068969000 \\ \mathrm{~F} & 3.636785000 \\ \mathrm{~F} & 4.110731000 \\ \mathrm{C} & -5.746335000 \\ \mathrm{C} & -4.470361000 \\ \mathrm{C} & -6.841207000 \\ \mathrm{H} & -7.834589000 \\ \mathrm{C} & -4.295936000 \\ \mathrm{C} & -6.671917000 \\ \mathrm{H} & -3.296663000 \\ \mathrm{H} & -7.530151000 \\ \mathrm{C} & -5.396809000 \\ \mathrm{H} & -5.254667000 \\ \mathrm{H} & -5.857753000 \\ \mathrm{~N} & -3.389458000 \\ \mathrm{C} & -2.240246000 \\ \mathrm{O} & -1.307706000 \\ \mathrm{H} & \end{array}$

4.062163000

3.544479000

2. 678740000

4.044650000

4. 250033000

2. 133443000

1.893312000

2. 591747000

1. 193154000

$-2.861617000$

$-2.892365000$

$-1.152740000$

$-0.772999000$

$-2.579768000$

$-2.287442000$

$-2.597512000$

$-2.006890000$

$-0.105702000$

$-0.553734000$

$-0.983112000$

1. 088781000

0.485905000

1.038003000

$-1.082876000$

$-0.051638000$

$-0.573019000$

$-0.906176000$

$-0.490902000$

$-1.957217000$

$-2.284314000$

$-2.363118000$

$-2.953019000$

$-2.803648000$

$-3.880841000$

1.028195000

0.333292000

0.028508000

$-0.599086000$

0.920257000

2. 212573000

2. 384159000

3. 179275000

1. 560405000

2.607287000

2. 322714000

3. 608155000

2.689230000

$-0.526374000$

$-2.667961000$

$-1.705987000$

$-2.602637000$

$-1.568710000$

1. 865720000

1. 147878000

3.593979000

2. 614776000

3.604890000

$-2.436555000$

$-2.286360000$

$-1.718185000$

0.383380000

0.227274000

$-0.025222000$

0.205720000

$-0.119884000$

$-0.297325000$

0.335896000

0.010179000

0.507694000

$-0.065069000$

0.235723000

0.335772000

$-0.126700000$

0.282979000

0.585097000

0.963624000

TS-1-Ge (PhNCO) : $E=-4895.762893$

$\mathrm{Ge}$

C

$\mathrm{H}$

$\mathrm{H}$

C

C

C

C

C
1. 306209000

0.638440000

1. 477505000

0.577141000

1.215541000

1. 418646000

3.306974000

3. 999177000

1.022445000

$-0.419757000$

$-0.957863000$

$-1.393611000$

$-0.459762000$

$-0.228015000$

0.587056000

$-0.586951000$

0.188768000

$-1.956572000$

$-1.200549000$

$-2.808459000$

$-2.296647000$
$-0.399268000$

1. 404280000

2.079009000

1. 341330000

$-1.866209000$

$-1.432215000$

0.045085000

0.293937000

$-1.461568000$

$-1.866885000$

2.124972000

3. 294778000

3. 051166000

4.108294000

3. 464111000

4.694470000

4.813408000

2.378966000

1. 679072000

1.789405000

2.997045000
$-0.038571000$

$-0.454238000$

$-0.231061000$

$-1.552966000$

1.376620000

2.834063000

0.071169000

$-1.271282000$

$-1.740233000$

$-2.064056000$

0.122541000

$-1.291858000$

1. 687205000

$-1.859307000$

$-2.221007000$

$-2.721170000$

$-1.129504000$

$-2.390946000$

$-2.778363000$

$-2.021643000$

$-3.237106000$ 


\begin{tabular}{|c|c|}
\hline C & -2.513594000 \\
\hline $\mathrm{H}$ & -2.926870000 \\
\hline $\mathrm{H}$ & -3.333118000 \\
\hline $\mathrm{H}$ & -2.148257000 \\
\hline $\mathrm{C}$ & 0.395117000 \\
\hline $\mathrm{H}$ & -0.168113000 \\
\hline $\mathrm{H}$ & 0.731336000 \\
\hline $\mathrm{H}$ & 1.296026000 \\
\hline $\mathrm{C}$ & -1.743475000 \\
\hline $\mathrm{H}$ & -2.349284000 \\
\hline $\mathrm{H}$ & -1.476559000 \\
\hline $\mathrm{H}$ & -2.368980000 \\
\hline $\mathrm{C}$ & 0.328677000 \\
\hline $\mathrm{H}$ & 1.328263000 \\
\hline $\mathrm{H}$ & 0.474291000 \\
\hline $\mathrm{H}$ & -0.215204000 \\
\hline $\mathrm{F}$ & -0.884761000 \\
\hline $\mathrm{F}$ & -0.486594000 \\
\hline $\mathrm{F}$ & -1.192402000 \\
\hline $\mathrm{F}$ & 1.417412000 \\
\hline $\mathrm{F}$ & 1.764951000 \\
\hline $\mathrm{F}$ & 0.132408000 \\
\hline $\mathrm{F}$ & 2.273684000 \\
\hline $\mathrm{F}$ & 1.907152000 \\
\hline $\mathrm{F}$ & 2.273874000 \\
\hline $\mathrm{F}$ & 0.268317000 \\
\hline $\mathrm{F}$ & 4.016531000 \\
\hline $\mathrm{F}$ & 3.333532000 \\
\hline $\mathrm{F}$ & 5.242303000 \\
\hline $\mathrm{F}$ & 3.393740000 \\
\hline $\mathrm{F}$ & 4.051784000 \\
\hline $\mathrm{C}$ & -5.822034000 \\
\hline $\mathrm{C}$ & -4.532842000 \\
\hline $\mathrm{C}$ & -6.914118000 \\
\hline $\mathrm{H}$ & -7.917999000 \\
\hline $\mathrm{C}$ & -4.341671000 \\
\hline $\mathrm{C}$ & -6.728677000 \\
\hline $\mathrm{H}$ & -3.332251000 \\
\hline $\mathrm{H}$ & -7.585072000 \\
\hline $\mathrm{C}$ & -5.440277000 \\
\hline $\mathrm{H}$ & -5.285856000 \\
\hline $\mathrm{H}$ & -5.945825000 \\
\hline $\mathrm{N}$ & -3.456540000 \\
\hline $\mathrm{C}$ & -2.294879000 \\
\hline 0 & -1.346390000 \\
\hline
\end{tabular}

4.225017000

4.770913000

3.655268000

4. 971309000

4. 298581000

5. 102313000

4.681682000

4.084064000

3. 409027000

2. 512410000

3.864335000

4.126935000

2.033644000

1. 842452000

2. 428657000

1.081261000

$-2.711310000$

$-2.434125000$

$-0.775911000$

$-0.671609000$

$-2.582733000$

$-2.647238000$

$-2.643786000$

$-2.416936000$

$-0.407072000$

$-1.041629000$

$-0.823170000$

1.226811000

0.710059000

1. 218865000

$-0.856740000$

$-0.089809000$

$-0.608733000$

$-0.949905000$

$-0.537271000$

$-1.995287000$

$-2.330456000$

$-2.398892000$

$-3.003193000$

$-2.847024000$

$-3.925960000$

0.992164000

0.305441000

$-0.001802000$

$-0.648875000$

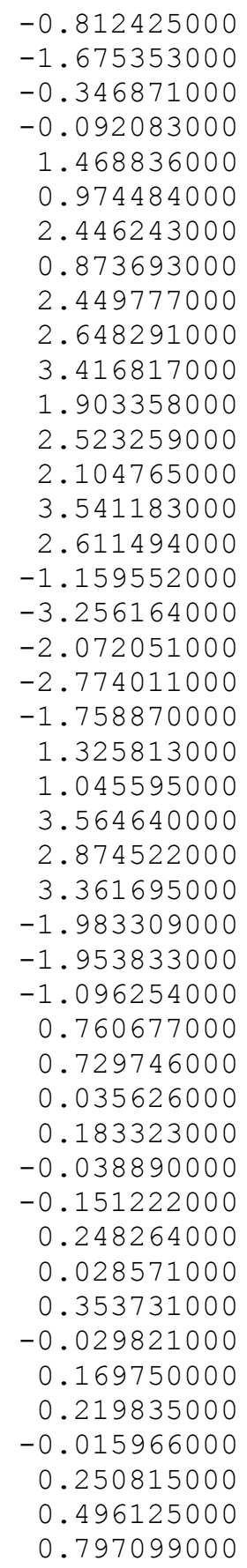

0.010182000

$-0.637516000$

$-0.430431000$

$-1.729376000$

1. 900539000

3.052731000

$-0.385330000$

$-1.855106000$

$-1.572789000$

$-1.613224000$

$-0.073586000$

$-1.569506000$

1. 381676000

$-2.265400000$ 


\begin{tabular}{|c|c|}
\hline $\mathrm{H}$ & 0.115568000 \\
\hline $\mathrm{H}$ & -1.244295000 \\
\hline $\mathrm{H}$ & -0.459037000 \\
\hline $\mathrm{C}$ & -2.231564000 \\
\hline $\mathrm{H}$ & -1.393446000 \\
\hline $\mathrm{H}$ & -3.002601000 \\
\hline $\mathrm{H}$ & -2.646960000 \\
\hline $\mathrm{C}$ & -3.028126000 \\
\hline $\mathrm{H}$ & -3.552087000 \\
\hline $\mathrm{H}$ & -3.730697000 \\
\hline $\mathrm{H}$ & -2.761715000 \\
\hline C & 0.018956000 \\
\hline $\mathrm{H}$ & -0.642336000 \\
\hline $\mathrm{H}$ & 0.354171000 \\
\hline $\mathrm{H}$ & 0.909818000 \\
\hline C & -2.000066000 \\
\hline $\mathrm{H}$ & -2.519960000 \\
\hline $\mathrm{H}$ & -1.760613000 \\
\hline $\mathrm{H}$ & -2.694840000 \\
\hline C & 0.191725000 \\
\hline $\mathrm{H}$ & 1.203368000 \\
\hline $\mathrm{H}$ & 0.308863000 \\
\hline $\mathrm{H}$ & -0.254086000 \\
\hline $\mathrm{F}$ & -1.032476000 \\
\hline $\mathrm{F}$ & -0.934239000 \\
\hline $\mathrm{F}$ & -1.635612000 \\
\hline $\mathrm{F}$ & 0.849946000 \\
\hline $\mathrm{F}$ & 1.487274000 \\
\hline $\mathrm{F}$ & 0.380091000 \\
\hline $\mathrm{F}$ & 2.347251000 \\
\hline $\mathrm{F}$ & 2.761703000 \\
\hline $\mathrm{F}$ & 2.996059000 \\
\hline $\mathrm{F}$ & 1.201355000 \\
\hline $\mathrm{F}$ & 3.637268000 \\
\hline $\mathrm{F}$ & 3.001531000 \\
\hline $\mathrm{F}$ & 5.044878000 \\
\hline $\mathrm{F}$ & 3.655541000 \\
\hline $\mathrm{F}$ & 4.200314000 \\
\hline $\mathrm{C}$ & -5.803634000 \\
\hline C & -4.499237000 \\
\hline $\mathrm{C}$ & -6.866875000 \\
\hline $\mathrm{H}$ & -7.884523000 \\
\hline C & -4.259277000 \\
\hline $\mathrm{C}$ & -6.634468000 \\
\hline $\mathrm{H}$ & -3.236518000 \\
\hline $\mathrm{H}$ & -7.468782000 \\
\hline $\mathrm{C}$ & -5.329387000 \\
\hline $\mathrm{H}$ & -5.139721000 \\
\hline $\mathrm{H}$ & -5.963805000 \\
\hline $\mathrm{N}$ & -3.446822000 \\
\hline C & -2.260011000 \\
\hline O & -1.222161000 \\
\hline
\end{tabular}

3.525439000

4.507424000

4.906485000

2. 050901000

1. 405950000

1. 410034000

2. 506564000

3. 959792000

4.321676000

3. 336645000

4.840143000

4.638450000

5.337039000

5.139023000

4.459596000

3.648192000

2. 722787000

4. 210113000

4. 257101000

2.483274000

2. 348731000

2. 979807000

1. 493717000

$-2.979712000$

$-3.136925000$

$-1.312354000$

$-1.123570000$

$-2.831718000$

$-2.051301000$

$-2.412815000$

$-1.475171000$

0.213075000

$-0.001248000$

$-1.169264000$

0.889802000

0.422182000

1. 289268000

$-0.824043000$

$-0.321706000$

$-0.792367000$

$-1.220553000$

$-0.848832000$

$-2.164231000$

$-2.587745000$

$-2.526746000$

$-3.289859000$

$-3.054201000$

$-4.122658000$

0.750542000

0.147953000

$-0.033309000$

$-0.541902000$
$-2.589663000$

$-3.166898000$

$-1.625401000$

$-2.538420000$

$-2.845151000$

$-2.086192000$

$-3.451312000$

$-1.146031000$

$-2.045131000$

$-0.570601000$

$-0.543129000$

1. 020192000

0.488123000

1. 943396000

0.398680000

2. 143248000

2.432889000

3. 060165000

1. 550255000

2. 291286000

1.876228000

3. 267419000

2. 480023000

$-0.492677000$

$-2.637800000$

$-1.734532000$

$-2.756091000$

$-1.539981000$

2.384591000

1. 528984000

3.884018000

2. 561481000

3. 732718000

$-2.375089000$

$-2.525837000$

$-2.020340000$

0.082794000

0.252293000

0.247022000

0.413021000

0.232132000

0.103818000

0.560040000

0.378216000

0.680544000

0.363692000

0.540324000

0.652761000

0.131884000

0.413487000

0.602107000

0.897205000

1.316919000

2. 270325000

1. 432714000

$-1.666068000$

$-1.130528000$

0.087732000

1.143818000 


\begin{tabular}{|c|c|c|c|}
\hline $\mathrm{C}$ & -0.248819000 & -4.355704000 & 0.211545000 \\
\hline $\mathrm{H}$ & 0.525095000 & -4.081916000 & -0.522344000 \\
\hline $\mathrm{H}$ & 0.133289000 & -5.198303000 & 0.810133000 \\
\hline $\mathrm{H}$ & -1.137392000 & -4.711457000 & -0.328174000 \\
\hline C & -1.668913000 & -3.563355000 & 2.135556000 \\
\hline $\mathrm{H}$ & -1.958348000 & -2.711758000 & 2.768980000 \\
\hline $\mathrm{H}$ & -2.569435000 & -3.949288000 & 1.643953000 \\
\hline $\mathrm{H}$ & -1.290417000 & -4.354898000 & 2.803721000 \\
\hline $\mathrm{C}$ & 0.709129000 & -2.846555000 & 1.934372000 \\
\hline $\mathrm{H}$ & 1.510315000 & -2.487898000 & 1.270825000 \\
\hline $\mathrm{H}$ & 0.522629000 & -2.087691000 & 2.709598000 \\
\hline $\mathrm{H}$ & 1.056648000 & -3.754860000 & 2.451968000 \\
\hline $\mathrm{C}$ & -2.342870000 & -1.979750000 & -1.120624000 \\
\hline C & -2.931845000 & -0.624039000 & -1.531490000 \\
\hline $\mathrm{H}$ & -3.490154000 & -0.147647000 & -0.713985000 \\
\hline $\mathrm{H}$ & -2.154477000 & 0.074251000 & -1.870046000 \\
\hline $\mathrm{H}$ & -3.634313000 & -0.786340000 & -2.364977000 \\
\hline $\mathrm{C}$ & -3.468027000 & -2.854739000 & -0.566833000 \\
\hline $\mathrm{H}$ & -4.293683000 & -2.879765000 & -1.296885000 \\
\hline $\mathrm{H}$ & -3.145657000 & -3.891462000 & -0.398869000 \\
\hline $\mathrm{H}$ & -3.872436000 & -2.455970000 & 0.376140000 \\
\hline $\mathrm{C}$ & -1.730370000 & -2.624728000 & -2.373054000 \\
\hline $\mathrm{H}$ & -1.238299000 & -3.583029000 & -2.161839000 \\
\hline $\mathrm{H}$ & -2.527212000 & -2.809437000 & -3.111483000 \\
\hline $\mathrm{H}$ & -0.991959000 & -1.951726000 & -2.833047000 \\
\hline $\mathrm{N}$ & 1.922450000 & -1.028152000 & -0.794215000 \\
\hline B & -1.199436000 & 1.091037000 & 0.940528000 \\
\hline $\mathrm{C}$ & 0.232867000 & 1.653387000 & 1.259452000 \\
\hline $\mathrm{C}$ & 0.526662000 & 3.024233000 & 1.124139000 \\
\hline $\mathrm{C}$ & 1.275680000 & 0.814757000 & 1.697730000 \\
\hline $\mathrm{C}$ & 1.798677000 & 3.526799000 & 1.392056000 \\
\hline $\mathrm{H}$ & -0.261343000 & 3.711875000 & 0.811370000 \\
\hline $\mathrm{C}$ & 2.552891000 & 1.304168000 & 1.956086000 \\
\hline $\mathrm{H}$ & 1.097206000 & -0.254024000 & 1.814121000 \\
\hline $\mathrm{C}$ & 2.816572000 & 2.665181000 & 1.800295000 \\
\hline $\mathrm{H}$ & 1.997382000 & 4.594144000 & 1.281222000 \\
\hline $\mathrm{H}$ & 3.347941000 & 0.622878000 & 2.264145000 \\
\hline $\mathrm{H}$ & 3.818477000 & 3.052681000 & 1.994743000 \\
\hline $\mathrm{C}$ & -2.288574000 & 2.033012000 & 0.309879000 \\
\hline $\mathrm{C}$ & -3.567489000 & 2.134851000 & 0.881530000 \\
\hline $\mathrm{C}$ & -2.031502000 & 2.773021000 & -0.857403000 \\
\hline $\mathrm{C}$ & -4.547085000 & 2.956556000 & 0.325450000 \\
\hline $\mathrm{H}$ & -3.803911000 & 1.565200000 & 1.784886000 \\
\hline $\mathrm{C}$ & -3.017244000 & 3.569593000 & -1.437077000 \\
\hline $\mathrm{H}$ & -1.050963000 & 2.690399000 & -1.333206000 \\
\hline C & -4.275208000 & 3.669317000 & -0.842067000 \\
\hline $\mathrm{H}$ & -5.528486000 & 3.034327000 & 0.796841000 \\
\hline $\mathrm{H}$ & -2.802722000 & 4.118200000 & -2.355850000 \\
\hline $\mathrm{H}$ & -5.044368000 & 4.301555000 & -1.289103000 \\
\hline $\mathrm{C}$ & 3.194676000 & -0.436633000 & -0.936968000 \\
\hline $\mathrm{C}$ & 4.316905000 & -1.225704000 & -0.659722000 \\
\hline $\mathrm{C}$ & 3.371118000 & 0.909227000 & -1.290188000 \\
\hline C & 5.595608000 & -0.680074000 & -0.737805000 \\
\hline $\mathrm{C}$ & 4.652760000 & 1.447289000 & -1.361676000 \\
\hline $\mathrm{C}$ & 5.770711000 & 0.658700000 & -1.088453000 \\
\hline $\mathrm{H}$ & 4.161800000 & -2.269020000 & -0.382004000 \\
\hline $\mathrm{H}$ & 2.497928000 & 1.535172000 & -1.486697000 \\
\hline $\mathrm{H}$ & 6.462422000 & -1.306386000 & -0.519736000 \\
\hline $\mathrm{H}$ & 4.776749000 & 2.498020000 & -1.629760000 \\
\hline $\mathrm{H}$ & 6.772748000 & 1.085535000 & -1.147877000 \\
\hline
\end{tabular}


3-Si (PhNCO): $E=-3108.448404$

$\mathrm{Si}$

C

$\mathrm{H}$

$\mathrm{H}$

C

C
1.066603000

0.745335000

1. 510783000

0.829774000

1.147089000

1.085433000

3. 023234000

3.936743000

1.169719000

$-0.150213000$

$-0.894986000$

$-1.706118000$

$-0.866758000$

$-0.752373000$

0.196435000

$-1.234100000$

$-0.530277000$

$-1.939017000$

$-1.009388000$

$-2.658165000$

$-2.344068000$

$-3.046960000$

$-3.559492000$

$-3.687076000$

$-2.912266000$

$-0.232608000$

$-0.879106000$

$-0.092039000$

0.754842000

$-2.278960000$

$-2.772683000$

$-2.186727000$

$-2.924635000$

$-0.011746000$

1. 027488000

0.013195000

$-0.448895000$

$-0.685229000$

0.048221000

$-1.017718000$

1. 632855000

2. 012292000

0.122914000

2.272438000

1. 555306000

1.793409000

$-0.173994000$

4. 094272000

3. 418692000

5.131953000

3.004949000

3.719985000

$-5.024642000$

$-3.653993000$

$-5.847310000$

$-6.910004000$

$-3.117426000$

$-5.311259000$

$-2.060707000$

$-5.951907000$
$-0.292697000$

1. 651676000

2.283442000

1. 777526000

$-2.034342000$

$-1.927648000$

0.159395000

0.622310000

$-0.948645000$

$-1.398265000$

2. 122810000

3. 263084000

2. 666744000

4.426900000

4.073783000

5.078322000

5.038570000

2.450213000

2.017582000

1. 637411000

3.131138000

3. 778802000

4. 309686000

2. 949159000

4.492843000

4.056044000

4.838058000

4.281835000

4. 107156000

2.658969000

1. 686066000

2.857106000

3. 434453000

1. 626243000

1.594655000

1.897918000

0.619165000

$-2.415958000$

$-1.750981000$

$-0.376445000$

0.039010000

$-1.995862000$

$-2.855276000$

$-2.706130000$

$-3.007644000$

$-0.872206000$

$-1.740699000$

$-0.344416000$

1. 689025000

0.966662000

1. 224697000

$-0.805011000$

$-0.715530000$

$-0.842286000$

$-1.834993000$

$-1.719731000$

$-2.118284000$

$-3.101318000$

$-2.238185000$

$-3.983801000$
$-0.048262000$

$-0.315861000$

0.144871000

$-1.406763000$

0.934032000

2. 465442000

0.411481000

$-0.730732000$

$-1.892126000$

$-2.535168000$

0.197090000

$-1.027636000$

1. 978838000

$-1.332923000$

$-1.761936000$

$-2.077962000$

$-0.448742000$

$-2.309124000$

$-2.704747000$

$-2.144906000$

$-3.072961000$

$-0.495196000$

$-1.311983000$

$-0.163577000$

0.329244000

2. 100363000

1.677717000

3.168242000

1. 617265000

2.579135000

2.450003000

3. 657783000

2.151540000

2.719770000

2.364000000

3.785990000

2.652091000

$-1.890826000$

$-3.797003000$

$-2.528365000$

$-2.713703000$

$-1.992524000$

0.583310000

0.619295000

3.062399000

2.883765000

2. 861603000

$-1.620830000$

$-1.346660000$

$-0.268599000$

1. 286318000

1.043869000

$-0.140797000$

0.132199000

$-0.189128000$

$-0.407685000$

0.376058000

0.047167000

0.601049000

0.013447000 


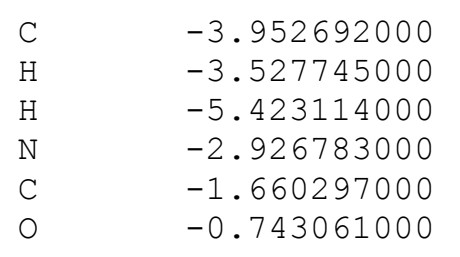

3-Ge (PhNCO): $E=-4895.795124$

\begin{tabular}{|c|c|c|c|}
\hline $\mathrm{Ge}$ & 1.098142000 & -0.277983000 & -0.049977000 \\
\hline C & 0.643512000 & 1.696820000 & -0.296293000 \\
\hline $\mathrm{H}$ & 1.381569000 & 2.336066000 & 0.200094000 \\
\hline $\mathrm{H}$ & 0.752522000 & 1.839247000 & -1.382462000 \\
\hline & 1.140991000 & -2.028635000 & 1.030978000 \\
\hline C & 1.093632000 & -1.867875000 & 2.554745000 \\
\hline $\mathrm{c}$ & 3.093389000 & 0.240993000 & 0.413676000 \\
\hline & 3.961046000 & 0.723778000 & -0.749227000 \\
\hline & 1.165116000 & -0.961823000 & -1.954105000 \\
\hline $\mathrm{C}$ & -0.159880000 & -1.442703000 & -2.557126000 \\
\hline & -1.028483000 & 2.092167000 & 0.194738000 \\
\hline & -1.832131000 & 3.226893000 & -1.041771000 \\
\hline & -1.010030000 & 2.650025000 & 1.973436000 \\
\hline & -0.894479000 & 4.405199000 & -1.339427000 \\
\hline & 0.064428000 & 4.068391000 & -1.758785000 \\
\hline & -1.379509000 & 5.047380000 & -2.090382000 \\
\hline & -0.691975000 & 5.021969000 & -0.454276000 \\
\hline & -2.038496000 & 2.407006000 & -2.323956000 \\
\hline $\mathrm{H}$ & -1.098584000 & 1.983602000 & -2.706323000 \\
\hline $\mathrm{H}$ & -2.750323000 & 1.586994000 & -2.164295000 \\
\hline $\mathrm{H}$ & -2.440860000 & 3.079988000 & -3.096284000 \\
\hline & -3.186967000 & 3.724820000 & -0.526631000 \\
\hline $\mathrm{H}$ & -3.693878000 & 4.253050000 & -1.348723000 \\
\hline $\mathrm{H}$ & -3.819435000 & 2.884786000 & -0.207678000 \\
\hline $\mathrm{H}$ & -3.072796000 & 4.437582000 & 0.302451000 \\
\hline C & -0.425877000 & 4.061333000 & 2.089279000 \\
\hline $\mathrm{H}$ & -1.099478000 & 4.818140000 & 1.663551000 \\
\hline 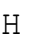 & -0.293853000 & 4.296153000 & 3.156407000 \\
\hline $\mathrm{H}$ & 0.559081000 & 4.147042000 & 1.606033000 \\
\hline 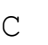 & -2.420063000 & 2.596222000 & 2.576396000 \\
\hline $\mathrm{H}$ & -2.873170000 & 1.601711000 & 2.466614000 \\
\hline $\mathrm{H}$ & -2.336956000 & 2.822318000 & 3.650377000 \\
\hline H & -3.097603000 & 3.333729000 & 2.130336000 \\
\hline c & -0.117023000 & 1.645034000 & 2.718894000 \\
\hline $\mathrm{H}$ & 0.924527000 & 1.652230000 & 2.366027000 \\
\hline $\mathrm{H}$ & -0.104334000 & 1.919295000 & 3.784688000 \\
\hline $\mathrm{H}$ & -0.517313000 & 0.623308000 & 2.650122000 \\
\hline $\mathrm{F}$ & -0.652538000 & -2.457927000 & -1.875714000 \\
\hline $\mathrm{F}$ & 0.014781000 & -1.816817000 & -3.816586000 \\
\hline $\mathrm{F}$ & -1.042410000 & -0.437536000 & -2.545614000 \\
\hline $\mathrm{F}$ & 1.587960000 & 0.047315000 & -2.766329000 \\
\hline $\mathrm{F}$ & 2.036566000 & -1.982050000 & -2.069007000 \\
\hline $\mathrm{F}$ & 0.131414000 & -2.857950000 & 0.692592000 \\
\hline $\mathrm{F}$ & 2.284347000 & -2.675943000 & 0.724404000 \\
\hline F & 1.577013000 & -2.924223000 & 3.182555000 \\
\hline $\mathrm{F}$ & 1.808269000 & -0.796443000 & 2.917923000 \\
\hline F & -0.159520000 & -1.672584000 & 2.955594000 \\
\hline F & 4.106256000 & -0.242001000 & -1.644042000 \\
\hline $\mathrm{F}$ & 3.385213000 & 1.772255000 & -1.345644000 \\
\hline $\mathrm{F}$ & 5.163035000 & 1.100650000 & -0.334910000 \\
\hline F & 3.030291000 & 1.302954000 & 1.282771000 \\
\hline $\mathrm{F}$ & 3.806724000 & -0.712745000 & 1.039941000 \\
\hline & -5.186415000 & -0.689725000 & -0.127511000 \\
\hline
\end{tabular}

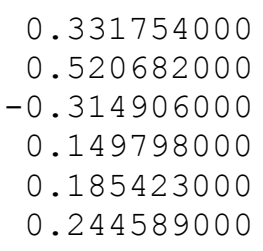

$\begin{array}{rr}-3.232820000 & 0.331754000 \\ -4.219942000 & 0.520682000 \\ 0.285149000 & -0.314906000 \\ 0.365291000 & 0.149798000 \\ 0.446820000 & 0.185423000 \\ -0.486011000 & 0.244589000\end{array}$

$-0.049977000$

00 .

.

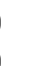




$\begin{array}{ll}\mathrm{C} & -3.809775000 \\ \mathrm{C} & -6.024421000 \\ \mathrm{H} & -7.090977000 \\ \mathrm{C} & -3.285516000 \\ \mathrm{C} & -5.499862000 \\ \mathrm{H} & -2.224214000 \\ \mathrm{H} & -6.152180000 \\ \mathrm{C} & -4.135793000 \\ \mathrm{H} & -3.717974000 \\ \mathrm{H} & -5.577783000 \\ \mathrm{~N} & -3.067662000 \\ \mathrm{C} & -1.792836000 \\ \mathrm{O} & -0.906741000\end{array}$

$$
\begin{array}{r}
-0.833298000 \\
-1.797929000 \\
-1.665110000 \\
-2.122675000 \\
-3.075352000 \\
-2.259077000 \\
-3.949300000 \\
-3.226280000 \\
-4.221687000 \\
0.317746000 \\
0.364968000 \\
0.401827000 \\
-0.530239000
\end{array}
$$

0.108540000
-0.170603000
-0.359097000
0.315397000
0.029811000
0.508324000
-0.001333000
0.274135000
0.434505000
-0.277512000
0.122232000
0.178716000
0.263083000

3-Sn (PhNCO): $E=-3033.263572$

\section{$\mathrm{Sn}$}

C

$\mathrm{H}$

$\mathrm{H}$

C

C

C

C

C

C
1.112473000

0.433646000

1. 147724000

0.476032000

1. 240757000

1.181608000

3.203443000

4.026933000

1.190595000

$-0.167136000$

$-1.238374000$

$-2.190929000$

$-1.158237000$

$-1.343711000$

$-0.406514000$

$-1.923323000$

$-1.101007000$

$-2.432600000$

$-1.498680000$

$-3.103424000$

$-2.900082000$

$-3.535649000$

$-4.115079000$

$-4.104577000$

$-3.405539000$

$-0.688910000$

$-1.443550000$

$-0.520200000$

0.258687000

$-2.522274000$

$-2.878775000$

$-2.406305000$

$-3.293140000$

$-0.144492000$

0.878350000

$-0.112602000$

$-0.453822000$

$-0.651549000$

$-0.083242000$

$-1.017776000$

1. 624164000

2.037854000

0.284360000

2.437903000

1.686317000

1.870504000
0.248673000

$-1.842582000$

$-2.535709000$

$-1.967118000$

2. 059206000

1. 808902000

$-0.477123000$

$-0.878171000$

1. 021329000

1.461376000

$-2.073516000$

$-3.187247000$

$-2.590819000$

$-4.426392000$

$-4.160283000$

$-5.067689000$

$-5.020530000$

$-2.389301000$

$-2.012727000$

$-1.539459000$

$-3.063702000$

$-3.598183000$

$-4.125331000$

$-2.717111000$

$-4.290679000$

$-4.043429000$

$-4.752702000$

$-4.260777000$

$-4.224812000$

$-2.403331000$

$-1.367689000$

$-2.646618000$

$-3.064093000$

$-1.651634000$

$-1.772476000$

$-1.888995000$

$-0.600212000$

2. 459039000

1. 841975000

0.428327000

0.021650000

2. 060360000

2. 962207000

2. 634636000

2.810147000

0.699292000
0.083755000

0.403906000

$-0.058521000$

1. 496590000

$-1.214542000$

$-2.722422000$

$-0.426383000$

0.790522000

2.156144000

2.702228000

$-0.177131000$

0.972937000

$-1.970377000$

1.295798000

1. 804400000

1.977249000

0.405154000

2. 263813000

2. 706120000

2.085971000

2. 997484000

0.363836000

1.137433000

0.036869000

$-0.479811000$

$-2.097989000$

$-1.730358000$

$-3.163725000$

$-1.568951000$

$-2.648381000$

$-2.567943000$

$-3.715614000$

$-2.234482000$

$-2.645184000$

$-2.258034000$

$-3.719353000$

$-2.557894000$

1. 983539000

3.967242000

2. 633367000

2.969687000

2.285859000

$-0.915968000$

$-0.951320000$

$-3.420571000$

$-3.017979000$ 


$\begin{array}{ll}\text { F } & -0.077429000 \\ F & 4.183906000 \\ \text { F } & 3.371314000 \\ \text { F } & 5.221194000 \\ \text { F } & 3.079108000 \\ \text { F } & 3.932890000 \\ \mathrm{C} & -5.325258000 \\ \mathrm{C} & -3.941328000 \\ \mathrm{C} & -6.152229000 \\ \mathrm{H} & -7.224371000 \\ \mathrm{C} & -3.399265000 \\ \mathrm{C} & -5.609418000 \\ \mathrm{H} & -2.331944000 \\ \mathrm{H} & -6.252795000 \\ \mathrm{C} & -4.238200000 \\ \mathrm{H} & -3.805970000 \\ \mathrm{H} & -5.731576000 \\ \mathrm{~N} & -3.213464000 \\ \mathrm{C} & -1.937406000 \\ \mathrm{O} & -1.042078000\end{array}$

$$
\begin{array}{r}
1.617681000 \\
0.171215000 \\
-1.825205000 \\
-1.350341000 \\
-1.609454000 \\
0.404002000 \\
0.788652000 \\
0.917634000 \\
1.905364000 \\
1.782315000 \\
2.203985000 \\
3.178982000 \\
2.329029000 \\
4.059746000 \\
3.316709000 \\
4.309121000 \\
-0.215355000 \\
-0.288441000 \\
-0.342133000 \\
0.572683000
\end{array}
$$

\begin{tabular}{|c|c|c|c|}
\hline $\mathrm{C}$ & 1.205279000 & 1.343766000 & 0.738101000 \\
\hline $\mathrm{H}$ & 1.401788000 & 1.356359000 & 1.818512000 \\
\hline $\mathrm{H}$ & 1.841671000 & 2.100530000 & 0.260372000 \\
\hline O & 0.032339000 & -0.750372000 & -0.242037000 \\
\hline $\mathrm{C}$ & -1.021461000 & -0.009644000 & -0.086255000 \\
\hline $\mathrm{P}$ & -0.523857000 & 1.688196000 & 0.458077000 \\
\hline $\mathrm{C}$ & -1.414041000 & 2.085096000 & 2.043579000 \\
\hline C & -2.882075000 & 2.438152000 & 1.785954000 \\
\hline $\mathrm{H}$ & -3.365457000 & 1.684862000 & 1.147185000 \\
\hline $\mathrm{H}$ & -3.407168000 & 2.466956000 & 2.753185000 \\
\hline & -2.983870000 & 3.430227000 & 1.324072000 \\
\hline C & -0.697377000 & 3.234365000 & 2.764093000 \\
\hline $\mathrm{H}$ & 0.344918000 & 2.981692000 & 3.005163000 \\
\hline & -0.704822000 & 4.164896000 & 2.181118000 \\
\hline $\mathrm{H}$ & -1.222387000 & 3.429196000 & 3.711972000 \\
\hline C & -1.332586000 & 0.810652000 & 2.900605000 \\
\hline $\mathrm{H}$ & -1.928203000 & -0.001114000 & 2.460375000 \\
\hline $\mathrm{H}$ & -0.298975000 & 0.454269000 & 3.032085000 \\
\hline $\mathrm{H}$ & -1.739948000 & 1.037659000 & 3.897608000 \\
\hline $\mathrm{C}$ & -0.783978000 & 2.834570000 & -0.986960000 \\
\hline C & 0.132074000 & 2.309914000 & -2.105337000 \\
\hline $\mathrm{H}$ & 1.198833000 & 2.362687000 & -1.848309000 \\
\hline $\mathrm{H}$ & -0.093272000 & 1.265181000 & -2.368605000 \\
\hline $\mathrm{H}$ & -0.034947000 & 2.928437000 & -3.000220000 \\
\hline $\mathrm{C}$ & -0.380634000 & 4.261067000 & -0.598294000 \\
\hline $\mathrm{H}$ & -0.375444000 & 4.884518000 & -1.505583000 \\
\hline $\mathrm{H}$ & -1.094832000 & 4.708964000 & 0.107679000 \\
\hline $\mathrm{H}$ & 0.627256000 & 4.302553000 & -0.158053000 \\
\hline $\mathrm{C}$ & -2.234899000 & 2.800862000 & -1.485109000 \\
\hline $\mathrm{H}$ & -2.937590000 & 3.237062000 & -0.765672000 \\
\hline $\mathrm{H}$ & -2.286335000 & 3.398723000 & -2.408172000 \\
\hline $\mathrm{H}$ & -2.561808000 & 1.777528000 & -1.711748000 \\
\hline $\mathrm{N}$ & -2.260323000 & -0.272112000 & -0.237492000 \\
\hline B & 1.439890000 & -0.217909000 & 0.101367000 \\
\hline $\mathrm{C}$ & 2.039805000 & -1.170677000 & 1.266232000 \\
\hline $\mathrm{C}$ & 3.415032000 & -1.225546000 & 1.541287000 \\
\hline $\mathrm{C}$ & 1.193735000 & -1.919816000 & 2.099804000 \\
\hline $\mathrm{C}$ & 3.922440000 & -1.974520000 & 2.602723000 \\
\hline $\mathrm{H}$ & 4.111556000 & -0.679638000 & 0.899408000 \\
\hline
\end{tabular}

3-B (PhNCO) : $E=-1582.147904$

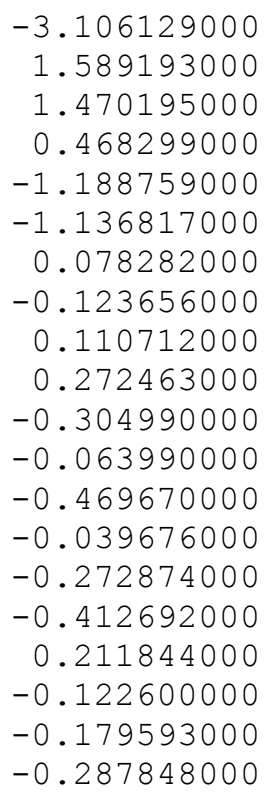

0.738101000

$-0.086255000$

.147185000

.753185000

(1)

2.181118000

3.711972000

3.032085000

3.897608000

$-1.848309000$

$-0.598294000$

$-1.485109000$

$-0.765672000$

4000

$-0.237492000$

1.266232000

1.541287000

2.602723000

0.899408000
$-1.505583000$ 
$-2.675092000$

$-1.928313000$

3.164049000

1. 902698000

$-2.701356000$

3.422899000

$-1.998504000$

2.786230000

$-3.252916000$

3. 788886000

$-3.293346000$

$-0.174269000$

4.251208000

$-1.258653000$

0.623157000

$-0.937902000$

$-1.377262000$

$-2.380081000$

$-2.550823000$

$-0.529973000$

$-3.561545000$

$-2.326901000$

$-3.652629000$

$-2.606170000$

$-4.417533000$

$-4.575099000$

$-0.612461000$

$-0.841404000$

$-0.750031000$

$-1.203274000$

$-1.106371000$

$-1.337369000$

$-0.724156000$

$-0.573637000$

$-1.378139000$

$-1.204357000$

$-1.617496000$ 